\title{
Numerical Taxonomy of Bacteria from the Gulf of Alaska
}

\author{
By JAMES D. HAUXHURST, ${ }^{+}$MICAH I. KRICHEVSKY ${ }^{2}$ \\ AND RONALD M. ATLAS ${ }^{1 *}$ \\ ${ }^{1}$ Department of Biology, University of Louisville, Louisville, Kentucky 40208, U.S.A. \\ ${ }^{2}$ Microbial Systematics Unit, National Institute of Dental Research, \\ National Institutes of Health, Bethesda, Maryland 20014, U.S.A.
}

(Received 7 November 1979; revised 11 March 1980)

\begin{abstract}
Numerical taxonomic analysis was performed on 247 bacterial strains isolated from the Northwest Gulf of Alaska in October 1975 and on 1010 bacterial strains isolated from the Northeast Gulf of Alaska in March 1976. Using the Jaccard similarity coefficient $\left(S_{J}\right)$ and single linkage clustering, 24 clusters (containing three or more strains) from the Northeast Gulf isolates and 12 clusters from the Northwest Gulf isolates were found at the $70 \%$ similarity level. The dominant organisms in the Northeast Gulf of Alaska were identified as being in the Moraxella-Acinetobacter group; these organisms were not found in the Northwest Gulf. The physiological and biochemical characteristics of the isolates in the clusters identified as belonging to the Moraxella-Acinetobacter group did not permit a clear separation of the two genera. Several clusters were tentatively identified as belonging to the Beneckea-Vibrio group from samples collected from both the Northeast and Northwest Gulf regions. A high proportion of the isolates from both regions were pigmented; several clusters of Flavobacterium occurred. Strains belonging to the genus Microcyclus were isolated from the Northwest Gulf but not from the Northeast Gulf of Alaska. Most clustered strains from the Northeast Gulf were eurytolerant, did not require growth factors and utilized a wide variety of substrates. Most isolates from the Northwest Gulf of Alaska were psychrotrophic and restricted to growth at salinities approximating that of seawater; many isolates from the Northwest Gulf demonstrated complex nutritional requirements. Many strains of Gram-negative pleomorphic rods isolated from both regions could not be identified.
\end{abstract}

\section{INTRODUCTION}

The ecology of micro-organisms in marine Alaskan continental shelf regions was studied as part of the U.S. Outer Continental Shelf Environmental Assessment Program. A major purpose of this undertaking has been to characterize the microbiological communities before mineral resources of the continental shelf are extensively developed. This study is concerned with the characterization of the predominant bacterial populations found in the Gulf of Alaska. Areas within the region studied have been considered as potential sites for offshore oil development and for development of oil transport facilities. If changes in biological communities are to be monitored, it is necessary to establish a baseline inventory of biomass, activities and species distribution through reconnaissance of the region prior to developmental activities. In order to establish the needed data base on the microbiological community, numerical taxonomic analyses were performed on bacterial isolates from surface

$\dagger$ Present address: University of Wisconsin Center, Fox Valley, Menasha, Wisconsin 54952, U.S.A. 


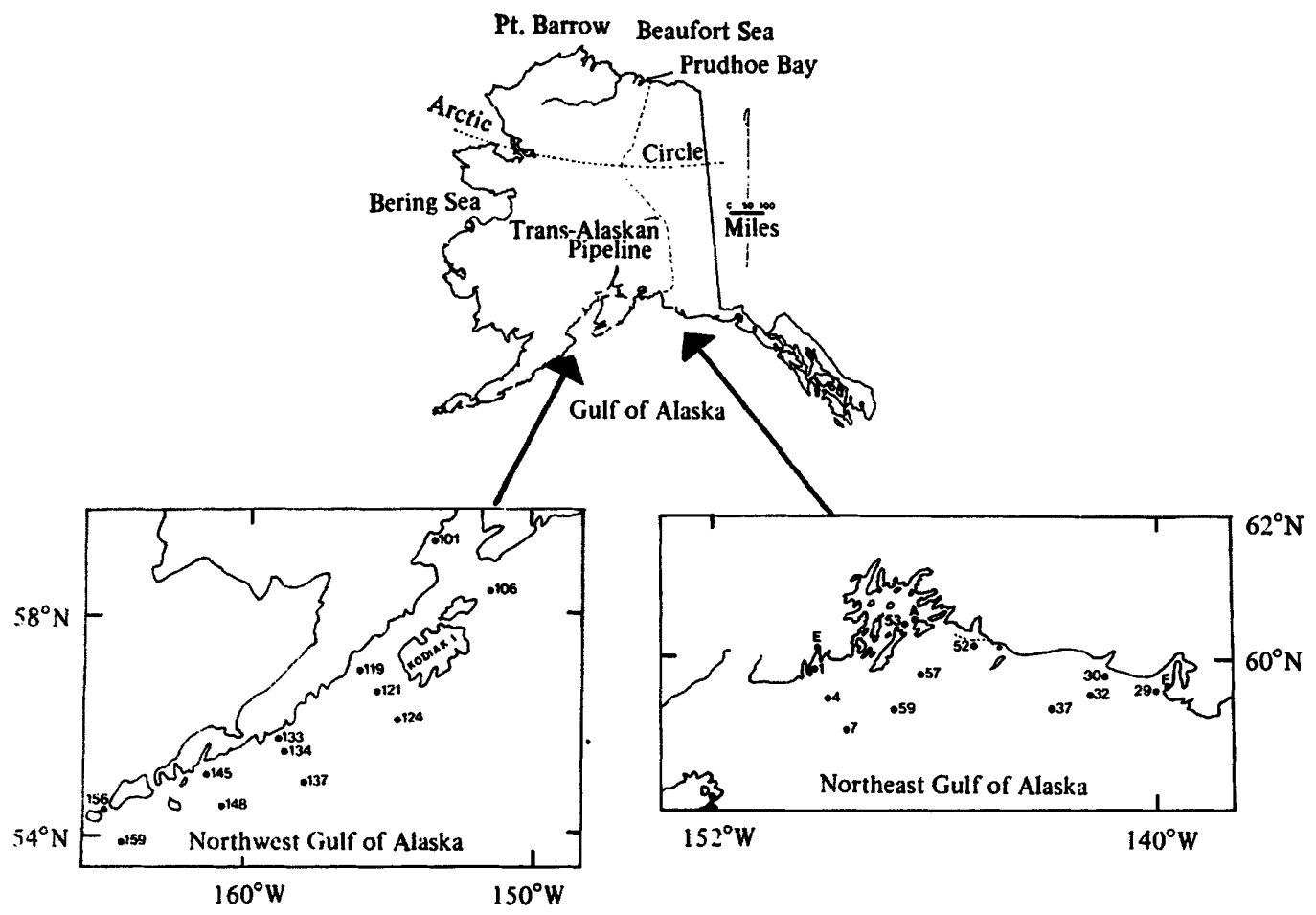

Fig. 1. Chart of sampling locations.

water and sediment collected from sites in the Northwest and Northeast regions of the Gulf of Alaska. A further aim was to determine whether bacterial populations are similar to those from other regions of the Pacific Ocean (ZoBell, 1946, 1961, 1963; ZoBell \& Upham, 1944; Simidu et al., 1977) or whether these populations from northern marine ecosystems possess adaptive features which distinguish them from populations isolated from temperate regions.

\section{METHODS}

Sample collection and bacterial isolation. Samples were collected in the Northwest Gulf of Alaska, between Unimak Pass and the mouth of Cook Inlet, during October 1975, and in the Northeast Gulf of Alaska, from Kodiak Island to Yakutat Bay, during March 1976 (Fig. 1). Surface water samples were collected with a sterile Niskin butterfly sampler (General Oceanics, Miami, Fla, U.S.A.) and sediment samples with a Van-Veen grab sampler (by courtesy of 1 . Kaplan). Samples were transferred to sterile containers stored at $5^{\circ} \mathrm{C}$. Enumeration (plating) procedures were initiated within $1 \mathrm{~h}$ of collection. Samples for bottom temperature and salinity determinations were collected with Nansen bottles (Wildco Instruments) with attached reversing thermometers. Intertidal sediment samples were collected directly with a sterile wide-mouth container.

Serial dilutions of samples were made in Rila marine salts solution (Rila Products, Teaneck, N.J., U.S.A.) and plated on marine agar 2216 (Difco). All materials were cooled to approximately $5^{\circ} \mathrm{C}$ before plating. For each sample, one set of replicate plates was incubated at $5{ }^{\circ} \mathrm{C}$ for 3 weeks and another at $20^{\circ} \mathrm{C}$ for 2 weeks. Colonies were counted with the aid of a Quebec colony counter; the mean count, from triplicate plates for sediment samples and sextuplicate plates for water samples, was recorded for each dilution and temperature.

All colonies were numbered sequentially; they were observed using a stereo microscope $(30 x)$ so that even relatively slowly growing bacteria were included in the pool of strains available for selection for isolation. Using random number tables, approximately 25 (from the Northwest Gulf cruise) to 60 (from the Northeast Gulf cruise) bacterial colonies from each sample were selected for isolation. After subculturing 
Table 1. Numbers and sources of isolates used in cluster analyses

\begin{tabular}{|c|c|c|c|c|}
\hline \multirow{2}{*}{$\begin{array}{c}\text { Station } \\
\text { (see Fig. 1) }\end{array}$} & \multicolumn{2}{|c|}{$5^{\circ} \mathrm{C}$ isolates } & \multicolumn{2}{|c|}{$20^{\circ} \mathrm{C}$ isolates } \\
\hline & Water & Sediment & Water & Sediment \\
\hline \multicolumn{5}{|c|}{ Northwest Gulf of Alaska } \\
\hline 101 & 8 & 18 & - & 10 \\
\hline 106 & 7 & - & - & - \\
\hline 119 & 6 & - & 5 & - \\
\hline 121 & - & 16 & - & 9 \\
\hline 124 & 12 & - & 10 & - \\
\hline 133 & 9 & - & 10 & - \\
\hline 134 & - & 19 & - & 7 \\
\hline 137 & 10 & 17 & 7 & 16 \\
\hline 145 & 11 & - & 5 & - \\
\hline 148 & 5 & - & 1 & - \\
\hline 156 & 12 & - & 6 & - \\
\hline 159 & 8 & - & 3 & - \\
\hline \multicolumn{5}{|c|}{ Northeast Gulf of Alaska } \\
\hline 1 & 30 & 22 & 35 & 35 \\
\hline 4 & 19 & 29 & 18 & 20 \\
\hline 7 & - & - & 27 & - \\
\hline 29 & - & - & 4 & - \\
\hline 30 & 17 & 31 & 8 & 31 \\
\hline 32 & 4 & 27 & 8 & 21 \\
\hline 37 & - & 25 & - & - \\
\hline 52 & 27 & 29 & 26 & 25 \\
\hline 53 & 24 & 22 & 7 & 27 \\
\hline 57 & 14 & 28 & 4 & 23 \\
\hline 59 & - & - & 10 & - \\
\hline $\mathbf{A}$ & 22 & 17 & 14 & \\
\hline D & 31 & 29 & 23 & 23 \\
\hline$\overline{\mathbf{E}}$ & 26 & - & 28 & - \\
\hline $\mathbf{F}$ & 28 & 3 & 20 & 33 \\
\hline
\end{tabular}

twice on marine agar plates to ensure purity and viability, about 25 strains from each sample were randomly selected for inclusion in the numerical phenetic analyses. Cultures were maintained on marine agar slants at $4^{\circ} \mathrm{C}$ and subcultured monthly. Some strains lost viability during testing and were discarded; more problems were found in maintaining the viability of isolates from the Northwest Gulf of Alaska than for those from the Northeast Gulf.

The following strains were successfully tested: 504 isolates from the $5^{\circ} \mathrm{C}$ Northeast Gulf enumeration plates, 506 isolates from the $20^{\circ} \mathrm{C}$ Northeast Gulf enumeration plates, 158 isolates from the $5{ }^{\circ} \mathrm{C}$ Northwest Gulf enumeration plates and 89 isolates from the $20^{\circ} \mathrm{C}$ Northwest Gulf enumeration plates. These isolates are designated ' $5{ }^{\circ} \mathrm{C}$ isolates' and ' $20^{\circ} \mathrm{C}$ isolates' depending on the plate incubation temperature. Their sources are shown in Table 1. Eleven ATCC reference strains, chosen to represent taxa associated with marine ecosystems, were included in the analyses: Alcaligenes pacificus 27122, Arthrobacter marinus 25374, Beneckea campbellii 25920, Flavobacterium halmephilum 19717, F. marinotypicum 19260, Pseudomonas coenobios 14402, P. doudoroffii 27123, P. marina 27129, P. nautica 27132, Vibrio alginolyticus 17749 and $V$. fischeri 15381.

Characterization of isolates. Approximately 300 phenotypic characteristics were determined for each strain. Details of the test procedures have been described (see Kaneko et al., 1979). Phenotypic characteristics examined included morphological examination of both cells and colonies, physiological growth ranges including tolerance to temperature, salt and $\mathrm{pH}$, biochemical tests including determination of a variety of enzymic activities, antibiotic sensitivity tests, and nutritional characteristics including the abilities to utilize a large number of biochemically diverse substrates (see Tables 4 and 6).

The test error rate, measured for repetitive testing of $5^{\circ} \mathrm{C}$ isolates included in this study, was approximately $3 \%$ at both 5 and $20^{\circ} \mathrm{C}$. Substrate utilization tests on 20 representative strains from clusters composed only of strains originally isolated at $20^{\circ} \mathrm{C}$ were repeated at $5{ }^{\circ} \mathrm{C}$ since identical organisms can show different test results at different incubation temperatures. Less than $3 \%$ of the repeated test results differed from the original results, which is within the range of error normally found for repetitive testing at a single temperature. 
Data processing and analysis. Data were coded and processed as previously described (Kaneko et al., 1979). Clustering was performed using the Jaccard coefficient $\left(S_{J}\right)$ (Sneath, 1957) and single linkage clustering (GTP2 program, by courtesy of R. R. Colwell). To minimize the formation of false clusters, an inherent problem with single linkage clustering, the data were checked prior to cluster analysis to ensure that no strains were included for which there were extensive missing data (i.e. $>30 \%$ ). Data could be missing due to loss of strain viability, contamination or other observable problems during testing. The $S_{J}$ coefficient was chosen because of the wide variety and large number of features determined, many of which gave numerous negative results for the diverse strains examined. At the time these analyses were performed, only programs with single linkage clustering algorithms were available. Since then, a portion of the data has been reanalysed using both weighted and unweighted average linkage with essentially identical results. Since all the data had been already analysed, it was decided to avoid the added expense of further analysis when the average linkage clustering algorithms became available.

Separate cluster analyses were performed for isolates from the Northeast and Northwest Gulf of Alaska. Because of the large number of strains included in the Northeast Gulf study, the initial cluster analyses of strains from this region were done using four independent subsets consisting of 242 strains isolated from water at $5{ }^{\circ} \mathrm{C}, 232$ strains isolated from water at $20^{\circ} \mathrm{C}, 262$ strains isolated from sediment at $5{ }^{\circ} \mathrm{C}$ and 274 strains isolated from sediment at $20^{\circ} \mathrm{C}$, respectively. These initial analyses yielded 85 clusters of two or more strains at about $70 \%$ similarity level; these clusters contained 346 strains. The data on strains occurring within these clusters were then pooled into a single combined set and re-run as another cluster analysis. A limitation of the combined cluster technique is that isolates which may be part of these clusters, but which occurred as single-membered clusters in the original four subsets, will have been left out of the analysis. There could be undetected clusters of four or less strains resulting from combination of single-membered clusters from the various subsets. Feature frequencies of all characteristics were determined with an updated version of the feature analysis program FREAK (Walczak, 1979).

Identification of defined phena. In addition to the inclusion of reference strains in the phenetic analyses, probabilistic identifications were attempted using the program IDDNEW and three identification matrices developed at the American Type Culture Collection (Johnson, 1979). Further attempts to identify phena to the genus level were performed using the taxonomic keys and the full text descriptions in Bergey's Manual of Determinative Bacteriology (Buchanan \& Gibbons, 1974) and the keys of Skerman (1967).

\section{RESULTS}

\section{Characteristics of samples}

The abiotic parameters - salinity, temperature and depth - and the viable bacterial counts found for samples collected from the Northwest and Northeast regions of the Gulf of Alaska are shown in Table 2. Temperatures at the time of sampling were higher in October when the Northwest region was sampled than in March when the Northeast region was sampled. Using viable plating enumeration procedures with incubation at both 5 and $20^{\circ} \mathrm{C}$, it was found that neither seasonal nor spatial variations were associated with corresponding fluctuations in viable counts.

\section{Characteristics and identification of Northeast Gulf of Alaska isolates}

Twenty-four clusters containing three or more strains were found at the $70 \%$ similarity level in the combined cluster analysis (performed after elimination of organisms that formed single-membered clusters in the four individual subset analyses) of isolates from the Northeast Gulf of Alaska. Seventeen clusters containing four or more isolates were considered to be major clusters. The largest cluster (E21) contained nearly equal numbers of isolates from water and sediment, all of which were isolated at $20^{\circ} \mathrm{C}$. Nine of the clusters contained strains from multiple sampling sites (Table 3), but generally the clusters did not contain strains isolated at different temperatures nor from both water and sediment samples. Indeed, only $12 \%$ of the clusters contained strains isolated both from water and from sediment. Clusters E4, E5, E6, E7, E8, E10, E11, E15, E16, E18, E19, E20, E22, E23 and E24 only contained isolates from plates incubated at $5^{\circ} \mathrm{C}$, and clusters E1, E3, E9, E12, E14, E17 and E21 only contained isolates from plates incubated at $20^{\circ} \mathrm{C}$.

The feature frequencies of the 17 major clusters are shown in Table 4. Most of the 
Table 2. Abiotic parameters and viable counts

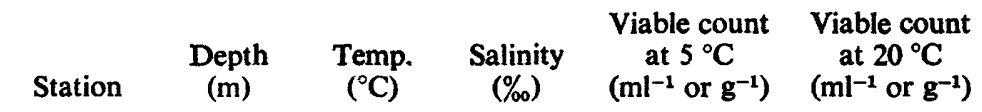

Water samples

$\begin{array}{llllll}101 & 0-2 & 9.1 & 30.8 & 5.5 \times 10^{1} & - \\ 106 & 0-2 & 7.7 & - & 6.5 \times 10^{1} & - \\ 119 & 0-2 & 8.6 & 29.6 & 3.5 \times 10^{1} & 4.3 \times 10^{1} \\ 124 & 0-2 & 8.3 & 31.8 & 9.7 \times 10^{1} & 8.0 \times 10^{1} \\ 133 & 0-2 & 8.8 & 30.8 & 9.2 \times 10^{1} & 1.1 \times 10^{2} \\ 137 & 0-2 & 8.6 & 31.3 & 1.2 \times 10^{2} & - \\ 145 & 0-2 & 8.0 & 31.4 & 1.3 \times 10^{2} & 3.1 \times 10^{2} \\ 148 & 0-2 & 7.8 & 31.9 & 1.9 \times 10^{2} & 5.7 \times 10^{2} \\ 156 & 0-2 & 6.4 & 31.7 & 2.5 \times 10^{2} & 2.0 \times 10^{3} \\ 159 & 0-2 & 7.5 & 31.9 & 2.2 \times 10^{2} & 5.6 \times 10^{2}\end{array}$

Sediment samples

$\begin{array}{lrcccc}101 & 91 & 9.3 & 30.8 & 1.0 \times 10^{4} & - \\ 121 & 230 & 4.7 & 33.5 & 1.1 \times 10^{6} & 8.4 \times 10^{5} \\ 134 & 154 & - & - & 1.4 \times 10^{6} & 1.2 \times 10^{8} \\ 137 & 99 & 7.7 & 31.9 & 6.2 \times 10^{5} & 3.5 \times 10^{5}\end{array}$

Water samples

\section{Northeast Gulf of Alaska}

$\begin{array}{llllll}1 & 0-2 & 3.0 & 31.7 & 3.3 \times 10^{1} & 3.9 \times 10^{1} \\ 4 & 0-2 & 3.5 & 32.2 & 2.1 \times 10^{1} & 2.0 \times 10^{1} \\ 7 & 0-2 & 3.5 & 32.3 & 3.1 \times 10^{2} & 2.2 \times 10^{2} \\ 29 & 0-2 & 4.2 & 32.0 & 1.5 \times 10^{1} & 1.8 \times 10^{1} \\ 30 & 0-2 & 3.8 & 31.7 & 3.3 \times 10^{1} & 3.2 \times 10^{1} \\ 32 & 0-2 & 4.5 & 32.2 & 1.2 \times 10^{1} & 5.8 \times 10^{1} \\ 52 & 0-2 & 3.2 & 31.8 & 7.2 \times 10^{1} & 1.0 \times 10^{2} \\ 53 & 0-2 & 3.0 & 31.8 & 5.9 \times 10^{1} & 2.6 \times 10^{2} \\ 57 & 0-2 & 4.0 & 32.1 & 1.0 \times 10^{1} & 2.7 \times 10^{1} \\ 59 & 0-2 & 4.0 & 32.2 & 1.4 \times 10^{1} & 1.7 \times 10^{2} \\ \text { A } & 0 & 5.0 & 31.1 & 7.7 \times 10^{2} & 2.9 \times 10^{3} \\ \text { D } & 0 & 2.0 & 31.4 & 2.2 \times 10^{4} & 2.0 \times 10^{4} \\ \text { E } & 0 & 3.0 & 31.1 & 1.1 \times 10^{3} & 9.9 \times 10^{2} \\ \text { F } & 0 & 3.0 & 30.8 & 3.8 \times 10^{2} & 3.8 \times 10^{2}\end{array}$

Sediment samples

$\begin{array}{rrrrrr}1 & 190 & 4 \cdot 3 & 32.4 & 5.2 \times 10^{5} & 2.1 \times 10^{5} \\ 4 & 183 & 4.6 & 32.8 & 1.1 \times 10^{5} & 5.5 \times 10^{4} \\ 30 & 40 & 4.2 & 31.9 & 5.8 \times 10^{3} & 1.2 \times 10^{5} \\ 32 & 175 & 5.8 & 33.2 & 1 \cdot 1 \times 10^{5} & 6.0 \times 10^{4} \\ 37 & 2282 & 2.1 & 34.6 & 3.7 \times 10^{3} & 3.3 \times 10^{3} \\ 52 & 46 & 3.7 & 31.9 & 1.7 \times 10^{5} & 4.7 \times 10^{5} \\ 53 & 303 & 4.9 & 32.5 & 3.0 \times 10^{6} & 6.1 \times 10^{8} \\ 57 & 71 & 4.3 & 32.3 & 4.2 \times 10^{5} & 3.1 \times 10^{5} \\ \text { A } & 0 & 5.0 & 31.1 & 4.0 \times 10^{6} & 1.7 \times 10^{7} \\ \text { D } & 0 & 2.0 & 31.4 & 1.8 \times 10^{5} & 2.4 \times 10^{5} \\ \text { F } & 0 & 3.0 & 30.8 & 1.5 \times 10^{3} & 4.4 \times 10^{3}\end{array}$

organisms grew within the range of temperatures and salt concentrations normally found in the sampling region. Strains in clusters E8, E9, E10, E11, E12, E13, E14, E15, E16, E17, E18, E19, E20, E21, E22 and E23 did not require growth factors; those in E6, E7 and E24 required vitamins; those recovered in E3, E4 and E5 required yeast extract plus Casamino acids; while those in E1 and E2 grew only on complex media.

None of the isolates formed defined clusters with the reference strains nor were any identified using the probabilistic identification matrices. 


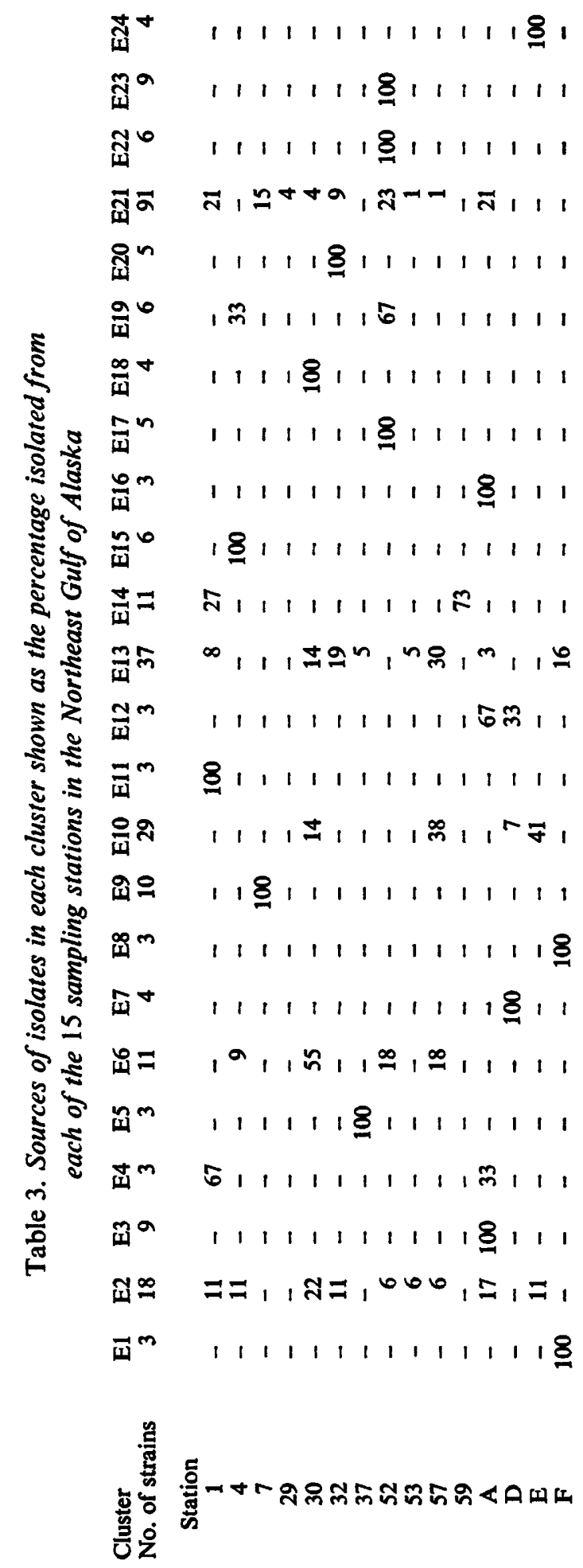


Table 4. Feature frequencies of selected characteristics of major clusters of bacterial populations isolated from the Northeast Gulf of Alaska*

Cluster

No. of strains

Cell morphology

Spherical

Rod-shaped

Curved axis

Coccobacillary

Pleomorphic

Cell length

$<2.0 \mu \mathrm{m}$

$>2.0 \mu \mathrm{m}$

Cell width

$0.5-1.0 \mu \mathrm{m}$

$1 \cdot 1-2 \cdot 0 \mu \mathrm{m}$

Cell arrangement

Single cells

Pairs

Miscellaneous cell features

Endospores produced

PHB $\uparrow$ inclusions

Capsule

Sudan black

Gram-positive

Gram-negative

Motile

Colony pigmentation

Non-diffusible pink

Non-diffusible brown

Non-diffusible yellow

Non-diffusible orange

White

Grey (no pigment)

Colony size

$<1 \mathrm{~mm}$

1-2 $\mathrm{mm}$

2-6 mm

Colony morphology

Translucent

Transparent

Opaque

Flat

Raised

Umbonate

Convex

Mucoid

Acid from

D-Ribose

D-Fructose

Cellobiose

Lactose

Sucrose

D-Mannitol

D-Glucose (aerobic)

D-Glucose (fermentative)

Miscellaneous features

Indole

Ammonia produced
E2 E3 E6 E7 E9 E10 E13 E14 E15 E17 E18 E19 E20 E21 E22 E23 E24

$\begin{array}{lllllllllllllllll}18 & 9 & 11 & 4 & 10 & 29 & 37 & 11 & 6 & 5 & 4 & 6 & 5 & 91 & 6 & 9 & 4\end{array}$

$\begin{array}{lllllllllllllllll}0 & 77 & 0 & 0 & 0 & 0 & 0 & 0 & 0 & 0 & 0 & 33 & 0 & 0 & 33 & 22 & 0\end{array}$

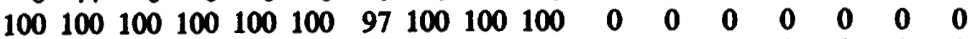
$\begin{array}{lllllllllllllllll}0 & 0 & 0 & 0 & 0 & 0 & 8 & 0 & 100 & 0 & 0 & 0 & 0 & 0 & 0 & 0 & 0\end{array}$

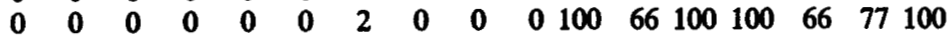

$\begin{array}{lllllllllllllllll}0 & 77 & 0 & 0 & 0 & 0 & 51 & 0 & 0 & 0 & 0 & 0 & 0 & 0 & 0 & 0 & 0\end{array}$

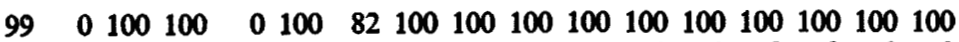
$\begin{array}{lllllllllllllllll}0 & 100 & 0 & 0 & 100 & 0 & 18 & 0 & 0 & 0 & 0 & 0 & 0 & 0 & 0 & 0 & 0\end{array}$

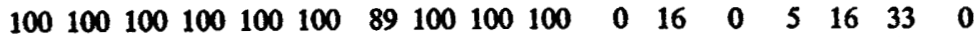

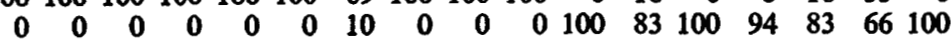

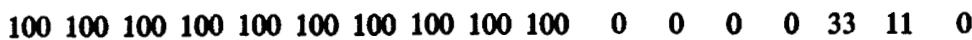
$\begin{array}{lllllllllllllllll}0 & 0 & 0 & 0 & 0 & 0 & 0 & 0 & 0 & 0 & 100 & 100 & 100 & 100 & 66 & 88 & 100\end{array}$

$\begin{array}{rrrrrrrrrrrrrrrrr}0 & 0 & 0 & 0 & 100 & 0 & 0 & 0 & 0 & 0 & 0 & 0 & 0 & 0 & 0 & 0 & 0 \\ 0 & 0 & 0 & 0 & 0 & 100 & 2 & 18 & 0 & 0 & 0 & 0 & 0 & 0 & 0 & 0 & 0 \\ 94 & 0 & - & - & 0 & - & 0 & 0 & - & - & - & - & - & 91 & - & - & - \\ 5 & 0 & 0 & 0 & 10 & 13 & 27 & 0 & 0 & 0 & 0 & 0 & 0 & 0 & 0 & 0 & 0 \\ 0 & 100 & 0 & 0 & 100 & 0 & 0 & 0 & 0 & 0 & 0 & 0 & 0 & 0 & 0 & 0 & 0\end{array}$ $\begin{array}{llllllllllllllllllllllll}100 & 0 & 100 & 100 & 0 & 100 & 100 & 100 & 100 & 100 & 100 & 100 & 100 & 100 & 100 & 100 & 100\end{array}$

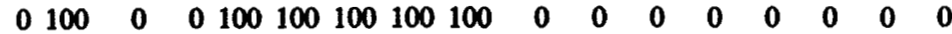

$\begin{array}{rrrrrrrrrrrrrrrrr}0 & 100 & 0 & 0 & 0 & 0 & 0 & 0 & 0 & 0 & 0 & 0 & 0 & 0 & 0 & 0 & 0 \\ 0 & 0 & 0 & 0 & 0 & 0 & 5 & 0 & 0 & 20 & 0 & 0 & 0 & 0 & 0 & 0 & 0 \\ 94 & 0 & 0 & 100 & 0 & 0 & 0 & 0 & 0 & 20 & 0 & 0 & 0 & 0 & 0 & 0 & 0 \\ 5 & 0 & 0 & 0 & 0 & 0 & 0 & 0 & 0 & 60 & 0 & 0 & 0 & 0 & 0 & 0 & 0 \\ 0 & 0 & 0 & 0 & 0 & 0 & 0 & 0 & 100 & 0 & 0 & 100 & 40 & 0 & 100 & 100 & 100 \\ 0 & 0 & 100 & 0 & 100 & 100 & 91 & 100 & 0 & 0 & 100 & 0 & 60 & 100 & 0 & 0 & 0\end{array}$

$\begin{array}{rrrrrrrrrrrrrrrrr}11 & 0 & 0 & 0 & 0 & 0 & 0 & 0 & 0 & 0 & 0 & 0 & 0 & 1 & 0 & 0 & 0\end{array}$ $\begin{array}{lllllllllllllllll}83 & 22 & 0 & 25 & 20 & 6 & 8 & 0 & 16 & 80 & 50 & 16 & 60 & 58 & 16 & 0 & 0\end{array}$

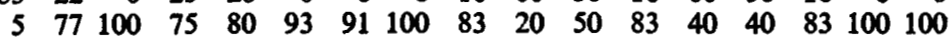

$\begin{array}{lllllllllllllllll}0 & 0 & 27 & 25 & 0 & 58 & 67 & 54 & 0 & 20 & 0 & 0 & 0 & 1 & 0 & 0 & 25\end{array}$

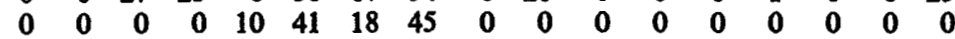
$\begin{array}{lllllllllllllllll}100 & 100 & 72 & 75 & 90 & 0 & 13 & 0 & 100 & 80 & 100 & 100 & 100 & 98 & 100 & 100 & 75\end{array}$ $\begin{array}{lllllllllllllllll}0 & 0 & 0 & 0 & 0 & 0 & 8 & 0 & 0 & 0 & 0 & 0 & 0 & 0 & 0 & 0 & 0\end{array}$

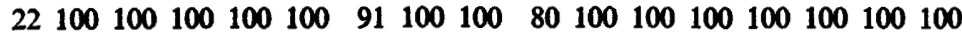
$\begin{array}{rrrrrrrrrrrrrrrrr}5 & 0 & 0 & 0 & 0 & 0 & 0 & 0 & 0 & 0 & 0 & 0 & 0 & 0 & 0 & 0 & 0 \\ 72 & 0 & 0 & 0 & 0 & 0 & 0 & 0 & 0 & 20 & 0 & 0 & 0 & 0 & 0 & 0 & 0\end{array}$ $\begin{array}{lllllllllllllllll}88 & 0 & 0 & 0 & 0 & 0 & 0 & 0 & 0 & 40 & 0 & 0 & 0 & 1 & 0 & 0 & 0\end{array}$

$\begin{array}{lllllllllllllllll}17 & 100 & 18 & 25 & 100 & 0 & 79 & 40 & 100 & 20 & 50 & 100 & 0 & 73 & 100 & 100 & 100\end{array}$

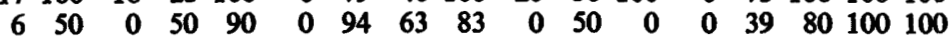

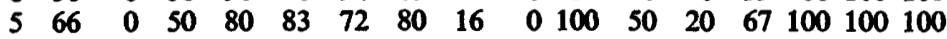

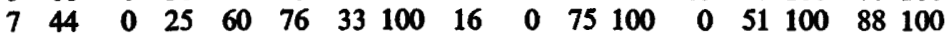

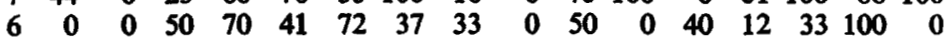
$\begin{array}{lllllllllllllllll}0 & 0 & 9 & 0 & 50 & 84 & 85 & 100 & 83 & 0 & 50 & 16 & 20 & 35 & 66 & 100 & 100\end{array}$ $\begin{array}{lllllllllllllllll}0 & 44 & 0 & 100 & 100 & 96 & 100 & 100 & 66 & 0 & 50 & 100 & 0 & 45 & 50 & 88 & 100\end{array}$

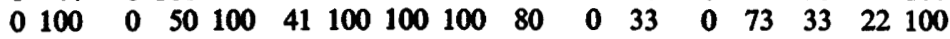

$\begin{array}{rrrrrrrrrrrrrrrrr}0 & 0 & 0 & 0 & 30 & 0 & 69 & 9 & 33 & 0 & 0 & 0 & 0 & 24 & 40 & 55 & 0\end{array}$ 
Table 4. (cont.)

Cluster

No. of strains

Nitrate reduced

Nitrite reduced

Gelatin hydrolysis

Starch hydrolysis

Tween 20 hydrolysis

Tween 80 hydrolysis

$\beta$-Haemolysis sheep blood

L-Arginine decarboxylase

L-Lysine decarboxylase

L-Ornithine decarboxylase

Alkaline phosphatase

Urease

Catalase

Oxidase

$\mathrm{pH}$ : growth at

pH 5

pH 6

pH 8

pH. 9

pH 10

Temperature: growth at

$$
5^{\circ} \mathrm{C}
$$

$20{ }^{\circ} \mathrm{C}$

$25^{\circ} \mathrm{C}$

$37^{\circ} \mathrm{C}$

$\mathrm{NaCl}$ : growth at

$$
0.5 \%(\mathrm{w} / \mathrm{v})
$$

$5.0 \%(w / v)$

$7.5 \%(w / v)$

$10.0 \%(\mathrm{w} / \mathrm{v})$

$15.0 \%(\mathrm{w} / \mathrm{v})$

Carbohydrates utilized

L-Arabinose

D-Ribose

D-Xylose

L-Rhamnose

D-Fructose

D-Galactose

D-Glucose

D-Mannose

L-Sorbose

Salicin

Cellobiose

Lactose

Maltose

Sucrose

Trehalose

Raffinose

Alcohols utilized

1-Butanol

Ethanol

1-Propanol

2-Propanol

$D(-)-1,2-P r o p a n e d i o l$

Glycerol

D-Arabitol

Dulcitol

D-Mannitol
E2 E3 E6 E7 E9 E10 E13 E14 E15 E17 E18 E19 E20 E21 E22 E23 E24

$\begin{array}{lllllllllllllllll}18 & 9 & 11 & 4 & 10 & 29 & 37 & 11 & 6 & 5 & 4 & 6 & 5 & 91 & 6 & 9 & 4\end{array}$

$\begin{array}{lllllllllllllllll}62 & 56 & 0 & 0 & 90 & 8 & 100 & 0 & 33 & 20 & 100 & 100 & 100 & 99 & 100 & 100 & 25\end{array}$

$\begin{array}{rrrrrrrrrrrrrrrrr}14 & 55 & 0 & 0 & 60 & 6 & 2 & 0 & 0 & 0 & 0 & 0 & 0 & 13 & 0 & 0 & 75\end{array}$

$\begin{array}{lllllllllllllllll}44 & 75 & 0 & 100 & 100 & 100 & 86 & 100 & 100 & 0 & 0 & 0 & 20 & 9 & 0 & 0 & 0\end{array}$

$\begin{array}{lllllllllllllllll}66 & 0 & 0 & 25 & 100 & 3 & 100 & 9 & 0 & 0 & 0 & 16 & 0 & 2 & 0 & 0 & 25\end{array}$

$\begin{array}{llllllllllllllllll}29 & 11 & 100 & 100 & 60 & 82 & 100 & 100 & 100 & 100 & 0 & 100 & 100 & 97 & 100 & 100 & 100\end{array}$

$\begin{array}{lllllllllllllllll}33 & 0 & 100 & 25 & 0 & 79 & 97 & 81 & 33 & 80 & 0 & 100 & 100 & 56 & 83 & 88 & 100\end{array}$

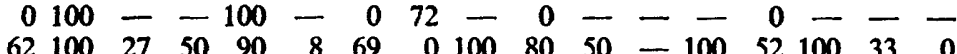
$\begin{array}{lllllllllllllllll}20 & 33 & 27 & 0 & 66 & 0 & 13 & 0 & 0 & 20 & 25 & 0 & 0 & 8 & 0 & 0 & 0\end{array}$ $\begin{array}{lllllllllllllllll}17 & 22 & 0 & 0 & 40 & 0 & 5 & 0 & 0 & 50 & 25 & 16 & 0 & 9 & 0 & 0 & 0\end{array}$

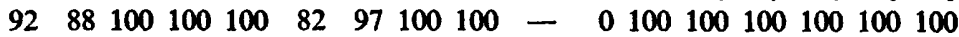
$\begin{array}{lllllllllllllllll}0 & 0 & 0 & 0 & 0 & 0 & 0 & 0 & 0 & 0 & 0 & 16 & 0 & 0 & 33 & 22 & 0\end{array}$ $\begin{array}{lllllllllllllllll}83 & 100 & 0 & 100 & 100 & 100 & 97 & 90 & 100 & 100 & 100 & 100 & 100 & 100 & 100 & 100 & 100\end{array}$

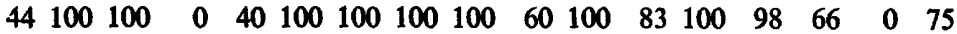

$\begin{array}{lllllllllllllllll}0 & 0 & 0 & 0 & 0 & 0 & 0 & 0 & 0 & 0 & 0 & 0 & 0 & 7 & 0 & 0 & 0\end{array}$ $\begin{array}{lllllllllllllllll}0 & 100 & 0 & 0 & 100 & 48 & 64 & 100 & 100 & 40 & 75 & 80 & 0 & 97 & 100 & 100 & 100\end{array}$

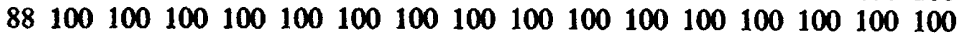

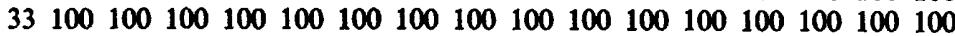
$\begin{array}{lllllllllllllllll}0 & 0 & 36 & 50 & 70 & 100 & 83 & 36 & 100 & 100 & 100 & 100 & 100 & 65 & 100 & 100 & 100\end{array}$

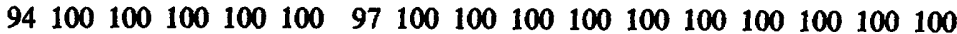

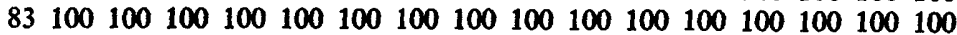
$\begin{array}{lllllllllllllllll}61 & 100 & 0 & 100 & 100 & 100 & 100 & 100 & 100 & 100 & 100 & 100 & 100 & 100 & 100 & 100 & 100\end{array}$

$\begin{array}{lllllllllllllllllllll}0 & 100 & 0 & 0 & 100 & 3 & 0 & 0 & 0 & 60 & 100 & 100 & 100 & 100 & 100 & 100 & 100\end{array}$

$\begin{array}{lllllllllllllllll}0 & 100 & 0 & 100 & 100 & 100 & 48 & 100 & 100 & 100 & 100 & 100 & 100 & 100 & 100 & 100 & 100\end{array}$ $\begin{array}{llllllllllllllllll}0 & 100 & 54 & 100 & 100 & 100 & 89 & 100 & 100 & 100 & 100 & 100 & 100 & 100 & 100 & 100 & 100\end{array}$ $\begin{array}{lllllllllllllllllll}0 & 100 & 0 & 100 & 100 & 100 & 5 & 100 & 100 & 100 & 100 & 100 & 100 & 100 & 100 & 100 & 100\end{array}$

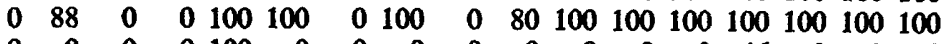
$\begin{array}{lllllllllllllllll}0 & 0 & 0 & 0 & 100 & 0 & 0 & 0 & 0 & 0 & 0 & 0 & 0 & 46 & 0 & 0 & 0\end{array}$

$\begin{array}{rrrrrrrrrrrrrrrrr}0 & 0 & 0 & 0 & 0 & 6 & 0 & 9 & 100 & 100 & 0 & 0 & 0 & 24 & 0 & 0 & 0 \\ 0 & 0 & 0 & 0 & 100 & 3 & 97 & 27 & 33 & 100 & 50 & 0 & 0 & 24 & 100 & 0 & 0 \\ 0 & 0 & 0 & 100 & 100 & 31 & 16 & 72 & 100 & 100 & 0 & 0 & 0 & 14 & 100 & 0 & 0 \\ 0 & 0 & 0 & 0 & 0 & 0 & 0 & 0 & 0 & 100 & 0 & 0 & 0 & 15 & 0 & 0 & 0 \\ 0 & 0 & 0 & 50 & 100 & 96 & 100 & 63 & 100 & 100 & 100 & 66 & 0 & 19 & 100 & 0 & 0 \\ 0 & 0 & 45 & 50 & 0 & 79 & 100 & 100 & 100 & 100 & 50 & 16 & 0 & 29 & 50 & 0 & 0 \\ 5 & 0 & 72 & 100 & 100 & 48 & 97 & 100 & 100 & 100 & 100 & 100 & 0 & 19 & 33 & 100 & 0 \\ 0 & 0 & 0 & 100 & 100 & 0 & 100 & 72 & 100 & 100 & 0 & 0 & 0 & 23 & 33 & 0 & 0 \\ 0 & 0 & 0 & 0 & 0 & 100 & 2 & 36 & 0 & 40 & 100 & 100 & 60 & 14 & 83 & 0 & 0 \\ 0 & 0 & 0 & 0 & 0 & 10 & 45 & 18 & 0 & 100 & 0 & 16 & 0 & 7 & 83 & 0 & 25 \\ 0 & 0 & 0 & 0 & 0 & 41 & 62 & 90 & 16 & 100 & 0 & 0 & 0 & 17 & 50 & 0 & 0 \\ 0 & 0 & 18 & 50 & 0 & 10 & 5 & 100 & 66 & 100 & 0 & 0 & 0 & 14 & 0 & 0 & 0 \\ 0 & - & 100 & 100 & 100 & 44 & 90 & 0 & 50 & 100 & 0 & 0 & 0 & 5 & 16 & 0 & 0 \\ 0 & 0 & 0 & 50 & 100 & 13 & 75 & 18 & 16 & 100 & 0 & 0 & 0 & 23 & 16 & 66 & 0 \\ 0 & 0 & 0 & 0 & 100 & 0 & 88 & 9 & 100 & - & 0 & 0 & 0 & 15 & 16 & 0 & 0 \\ 0 & 0 & 0 & 0 & 0 & 0 & 2 & 36 & 0 & 100 & 0 & 0 & 0 & 19 & 0 & 0 & 0\end{array}$

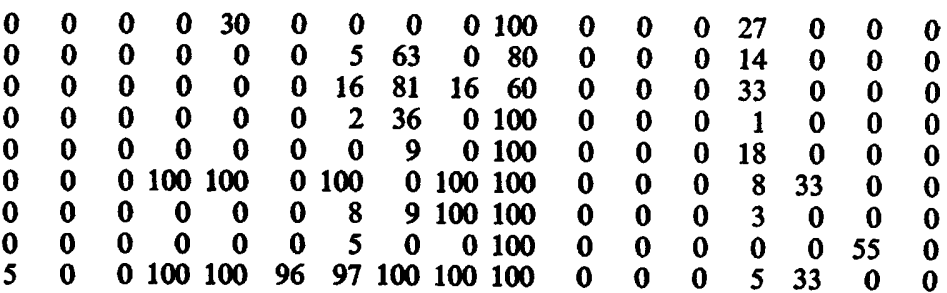


Table 4. (cont.)

Cluster

No. of strains

D-Sorbitol

meso-Inositol

Phenol

2-Phenyl ethanol

Carboxylic acids utilized

Acetic acid

Butyric acid

Caproic acid

Caprylic acid

Lauric acid

Propionic acid

Valeric acid

Glutaric acid

Malonic acid

Succinic acid

Oleic acid

Fumaric acid

Itaconic acid

DL-Glyceric acid

$\beta$-Hydroxybutyric acid DL-Lactic acid

$\mathbf{L}(+)$-Tartaric acid

Citric acid

2-Ketogluconic acid

Pyruvic acid

2-Ketoglutaric acid

Benzoic acid

$m$-Hydroxybenzoic acid

p-Hydroxybenzoic acid

Ascorbic acid

Galacturonic acid

D-Gluconic acid

o-Hydroxybenzoic acid

Stearic acid

Amino acids utilized

L-Alanine

$\gamma$-Aminobutyric acid

L-Arginine

L-Asparagine

L-Aspartic acid

L-Cysteine

L-Cystine

L-Glutamic acid

Glycine

L-Leucine

L-Isoleucine

L-Lysine

L-Methionine

L-Ornithine

L-Phenylalanine

L-Proline

L-Serine

L-Threonine

L-Tryptophan

L-Tyrosine

L-Valine

Amines utilized

Histamine

Putrescine
E2 E3 E6 E7 E9 E10 E13 E14 E15 E17 E18 E19 E20 E21 E22 E23 E24

$\begin{array}{lllllllllllllllll}18 & 9 & 11 & 4 & 10 & 29 & 37 & 11 & 6 & 5 & 4 & 6 & 5 & 91 & 6 & 9 & 4\end{array}$

$\begin{array}{rrrrrrrrrrrrrrrrr}0 & 0 & 0 & 100 & 10 & 0 & 18 & 0 & 0 & 100 & 0 & 0 & 0 & 3 & 0 & 0 & 0 \\ 0 & 0 & 0 & 0 & 0 & 0 & 78 & 0 & 0 & 100 & 0 & 0 & 0 & 2 & 33 & 0 & 0 \\ 0 & 0 & 0 & 0 & 0 & 0 & 0 & 9 & 0 & 100 & 0 & 0 & 0 & 2 & 0 & 0 & 0 \\ 0 & 0 & 0 & 0 & 0 & 0 & 0 & 0 & 0 & 100 & 0 & 0 & 0 & 0 & 0 & 0 & 0\end{array}$

\begin{tabular}{|c|c|c|c|c|c|c|c|c|c|c|c|c|c|c|c|}
\hline 0 & 0 & & 100 & 0 & 54 & 100 & 66 & 100 & 0 & 16 & 40 & 81 & & 00 & \\
\hline 0 & 0 & & 100 & 0 & 21 & 45 & 100 & 100 & 0 & 50 & 0 & 97 & 50 & 77 & \\
\hline 0 & 0 & & 100 & 13 & 86 & 72 & 100 & 100 & & 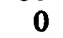 & & 100 & 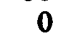 & 11 & \\
\hline 0 & 0 & & 100 & 0 & 73 & 100 & 100 & 100 & 0 & 0 & 0 & 92 & 0 & 0 & \\
\hline 0 & 0 & 0 & 0 & 0 & 13 & 0 & & 100 & & 0 & 0 & 7 & 16 & 66 & \\
\hline- & 0 & 0 & - & 17 & 0 & - & 100 & - & 0 & 0 & 0 & - & 0 & 0 & \\
\hline 33 & 0 & 0. & 100 & 0 & 64 & 72 & 100 & 100 & & 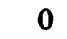 & 100 & 64 & 66 & 55 & \\
\hline 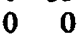 & 0 & 0 & & 0 & 18 & 0 & & 100 & & 0 & 0 & 83 & 0 & 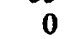 & \\
\hline 0 & 0 & 0 & 0 & 0 & 0 & 63 & 0 & 100 & & 0 & 0 & 4 & 0 & 0 & \\
\hline - & 0 & 0 & - & 51 & 85 & - & 100 & - & & 0 & 0 & - & 83 & 0 & \\
\hline 0 & 0 & 0 & 0 & 0 & 0 & 18 & 0 & 100 & & 0 & 0 & 0 & 0 & 0 & \\
\hline 0 & 9 & & 100 & 10 & 97 & 100 & 100 & 10 & 0 & 66 & 40 & 64 & 33 & 77 & \\
\hline 0 & 0 & 0 & 0 & 0 & 5 & o & 0 & 100 & 0 & 0 & 0 & 3 & 0 & 0 & \\
\hline 0 & - & -1 & 100 & - & 100 & 36 & - & 100 & & - & - & 18 & - & - & \\
\hline 0 & 0 & 0 & 0 & 0 & 13 & 27 & 100 & 100 & 0 & 0 & 0 & 60 & 0 & 0 & \\
\hline 0 & 0 & 0 & 0 & 0 & 100 & 27 & 100 & 100 & 75 & 83 & 100 & 82 & 100 & 55 & \\
\hline 0 & 0 & 0 & 0 & 34 & 0 & 9 & 0 & 10 & $\mathbf{0}$ & 33 & 0 & 34 & 50 & 77 & 10 \\
\hline 0 & 0 & & 100 & 13 & 91 & 18 & 100 & 100 & 50 & 100 & 0 & 35 & 50 & 0 & \\
\hline 0 & 0 & & 100 & 82 & 5 & 0 & 100 & 10 & & 100 & 0 & 24 & 33 & 0 & \\
\hline 0 & 9 & 0 & 100 & 100 & 83 & 100 & 100 & 100 & 50 & 83 & 60 & 100 & 100 & 44 & \\
\hline 0 & 0 & & 100 & 75 & 56 & 72 & 100 & 1 & 100 & 100 & 60 & 80 & 33 & 100 & \\
\hline 0 & 0 & 0 & 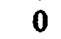 & 0 & 13 & 0 & 0 & & 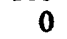 & 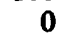 & 0 & 65 & 33 & 100 & \\
\hline 0 & 0 & 0 & 0 & 8 & 0 & 0 & & 100 & & 0 & 0 & 3 & 0 & 0 & \\
\hline 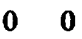 & 0 & 0 & 0 & 0 & 2 & 0 & 83 & 10 & & 0 & & & 50 & 0 & \\
\hline 0 & 0 & 0 & 0 & 58 & 16 & 36 & 0 & & & 0 & & 6 & 0 & 11 & \\
\hline 0 & & 50 & 0 & 0 & 5 & & 100 & 100 & & 0 & & 9 & 0 & & \\
\hline o & 0 & & 100 & 41 & 2 & 18 & 100 & 40 & 50 & 66 & 0 & 25 & 16 & 0 & \\
\hline & & 0 & 10 & & 2 & 0 & & & 10 & 33 & 60 & 22 & 56 & 22 & \\
\hline ( & & 0 & 0 & & 0 & 0 & & & & 0 & 0 & 3 & 0 & 0 & \\
\hline
\end{tabular}

$\begin{array}{lllllllllllllllll}0 & 0 & 0 & 0 & 0 & 24 & 67 & 100 & 100 & 100 & 0 & 0 & 0 & 78 & 0 & 0 & 0\end{array}$

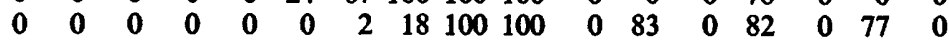
$\begin{array}{lllllllllllllllll}0 & 0 & 0 & 0 & 70 & 0 & 64 & 36 & 100 & 60 & 0 & 0 & 60 & 53 & 33 & 0 & 0\end{array}$ $\begin{array}{lllllllllllllllll}0 & 0 & 0 & 0 & 0 & 3 & 64 & 90 & 100 & 40 & 100 & 100 & 60 & 72 & 100 & 100 & 0\end{array}$ $\begin{array}{lllllllllllllllll}0 & 11 & 0 & 100 & 100 & 0 & 83 & 100 & 100 & 100 & 0 & 0 & 0 & 35 & 50 & 11 & 0\end{array}$

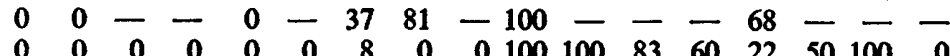
$\begin{array}{lllllllllllllllll}0 & 0 & 0 & 0 & 60 & 58 & 97 & 63 & 100 & 100 & 0 & 0 & 0 & 42 & 66 & 22 & 25\end{array}$ $\begin{array}{lllllllllllllllll}0 & 0 & 0 & 0 & 0 & 0 & 72 & 63 & 0 & 0 & 0 & 0 & 0 & 6 & 33 & 0 & 0\end{array}$ $\begin{array}{lllllllllllllllll}0 & 0 & 0 & 0 & 0 & 3 & 8 & 0 & 100 & 100 & 0 & 0 & 0 & 76 & 83 & 0 & 0\end{array}$ $\begin{array}{rrrrrrrrrrrrrrrrr}0 & 0 & 27 & 0 & 0 & 0 & 2 & 54 & 100 & 80 & 0 & 66 & 60 & 14 & 50 & 22 & 0\end{array}$ $\begin{array}{lllllllllllllllll}0 & 0 & 0 & 0 & 0 & 0 & 12 & 36 & 33 & 0 & 0 & 66 & 60 & 80 & 0 & 0 & 0\end{array}$ $\begin{array}{llllllllllllllllll}0 & 0 & 0 & 0 & 0 & 0 & 5 & 27 & 0 & 20 & 0 & 0 & 0 & 27 & 0 & 0 & 0\end{array}$ $\begin{array}{lllllllllllllllll}0 & 0 & 0 & 0 & 100 & 0 & 78 & 9 & 66 & 100 & 0 & 100 & 40 & 53 & 33 & 0 & 0\end{array}$ $\begin{array}{lllllllllllllllll}0 & 0 & 0 & 0 & 0 & 0 & 10 & 72 & 16 & 60 & 0 & 0 & 0 & 82 & 50 & 33 & 0\end{array}$ $\begin{array}{lllllllllllllllll}0 & 0 & 0 & 0 & 100 & 0 & 100 & 81 & 100 & 100 & 0 & 0 & 40 & 93 & 83 & 11 & 0\end{array}$ $\begin{array}{lllllllllllllllll}0 & 0 & 0 & 0 & 20 & 10 & 83 & 81 & 50 & 100 & 0 & 0 & 0 & 60 & 0 & 0 & 0\end{array}$ $\begin{array}{lllllllllllllllll}0 & 0 & 0 & 0 & 0 & 0 & 94 & 72 & 0 & 100 & 0 & 0 & 0 & 5 & 0 & 0 & 0\end{array}$ $\begin{array}{lllllllllllllllll}0 & 0 & 0 & 0 & 0 & 0 & 2 & 54 & 0 & 40 & 0 & 0 & 0 & 30 & 0 & 11 & 0\end{array}$ $\begin{array}{lllllllllllllllll}0 & 0 & 0 & 0 & 0 & 0 & 14 & 63 & 0 & 80 & 0 & 0 & 0 & 100 & 0 & 100 & 0\end{array}$ $\begin{array}{llllllllllllllllll}0 & 0 & 0 & 0 & 0 & 0 & 0 & 27 & 0 & 60 & 0 & 0 & 0 & 9 & 0 & 0 & 0\end{array}$

$\begin{array}{lllllllllllllllll}0 & 0 & 0 & 0 & 0 & 0 & 0 & 63 & 0 & 100 & 0 & 0 & 0 & 14 & 0 & 0 & 0\end{array}$ 
Table 4. (cont.)

Cluster

No. of strains

$\begin{array}{rrrrrrrrrrrrrrrrr}\text { E2 } & \text { E3 } & \text { E6 } & \text { E7 } & \text { E9 } & \text { E10 } & \text { E13 } & \text { E14 } & \text { E15 } & \text { E17 } & \text { E18 } & \text { E19 } & \text { E20 } & \text { E21 } & \text { E22 } & \text { E23 } & \text { E24 } \\ 18 & 9 & 11 & 4 & 10 & 29 & 37 & 11 & 6 & 5 & 4 & 6 & 5 & 91 & 6 & 9 & 4 \\ 0 & 0 & 54 & 0 & 100 & 0 & 94 & 36 & 100 & 80 & 0 & 0 & 0 & 17 & 50 & 0 & 25 \\ 0 & 0 & 0 & 0 & 0 & 0 & 0 & 36 & 0 & 100 & 0 & 0 & 0 & 4 & 0 & 0 & 0 \\ 0 & 0 & 0 & 0 & 0 & 0 & 2 & 18 & 0 & 100 & 0 & 0 & 0 & 8 & 0 & 0 & 0\end{array}$

$N$-Acetylglucosamine

Guanine

Thymine

Hydrocarbons utilized $n$-Hexadecane

2-Methylnaphthalene

$\omega$-Phenyldecane

Pristane

Pentadecylcyclohexane

$\begin{array}{lllllllllllllllll}\mathbf{0} & \mathbf{0} & \mathbf{0} & \mathbf{0} & \mathbf{0} & \mathbf{0} & \mathbf{5} & \mathbf{0} & \mathbf{0} & 100 & \mathbf{0} & \mathbf{0} & \mathbf{0} & \mathbf{9} & \mathbf{0} & \mathbf{0} & \mathbf{0} \\ \mathbf{0} & \mathbf{0} & \mathbf{0} & \mathbf{0} & \mathbf{0} & \mathbf{0} & \mathbf{0} & \mathbf{0} & \mathbf{0} & \mathbf{1 0 0} & \mathbf{0} & \mathbf{0} & \mathbf{0} & \mathbf{0} & \mathbf{0} & \mathbf{0} & \mathbf{0} \\ \mathbf{0} & \mathbf{0} & \mathbf{0} & \mathbf{0} & \mathbf{0} & \mathbf{0} & \mathbf{0} & \mathbf{0} & \mathbf{0} & \mathbf{1 0 0} & \mathbf{0} & \mathbf{0} & \mathbf{0} & \mathbf{0} & \mathbf{0} & \mathbf{0} & \mathbf{0} \\ \mathbf{0} & \mathbf{0} & \mathbf{0} & \mathbf{0} & \mathbf{0} & \mathbf{0} & \mathbf{0} & \mathbf{0} & \mathbf{0} & 100 & \mathbf{0} & \mathbf{0} & \mathbf{0} & \mathbf{2} & \mathbf{0} & \mathbf{6} & \mathbf{0} \\ \mathbf{0} & \mathbf{0} & \mathbf{0} & \mathbf{0} & \mathbf{0} & \mathbf{0} & \mathbf{2} & \mathbf{9} & \mathbf{0} & \mathbf{1 0 0} & \mathbf{0} & \mathbf{0} & \mathbf{0} & \mathbf{4} & \mathbf{0} & \mathbf{0} & \mathbf{0}\end{array}$

* The following features were $100 \%$ positive for all strains: rounded ends, entire colonies, glistening colonies, smooth colonies, facultative anaerobe, growth at pH 7, growth at $10{ }^{\circ} \mathrm{C}$, growth at $15^{\circ} \mathrm{C}$ and growth at $3.0 \% \mathrm{NaCl}$.

+ PHB, Poly- $\beta$-hydroxybutyric acid.

Based on the features shown in Table 4, six categories of the major clusters can be described:

1. Gram-negative, non-motile rods-coccobacilli often occurring as pairs (clusters E18 to E24). The morphological and metabolic features of the organisms closely resembled those of the Acinetobacter-Moraxella group as described in Bergey's Manual of Determinative Bacteriology (Lautrop, 1974). The genera Acinetobacter and Moraxella are distinguished by the oxidase test, strains of the former being negative while those of the latter are positive (Baumann et al., 1968; Lautrop, 1974). Additionally, strains of the genus Acinetobacter utilize carbohydrates, may form acid from sugar and are resistant to penicillin; strains of the genus Moraxella do not utilize carbohydrates and are sensitive to penicillin (Lautrop, 1974; Thornley, 1967; Shewan, 1971). In the present study, strains in cluster E23 meet all the above characteristics of the genus Acinetobacter and strains in cluster E20 all those of Moraxella. The other clusters (E18, E19, E21, E22 and E24) represent intermediate cases which could be classified as either Acinetobacter or Moraxella depending on which of the above criteria were used.

2. Gram-negative, motile, oxidase-positive, fermentative, curved or straight rods (clusters E10, E13, E14 and E15). Members of these clusters resembled strains classified in the genera Aeromonas, Beneckea and Vibrio; the distinction between these taxa is not clear (Baumann et al., 1971).

3. Gram-negative, non-motile rods producing yellow, orange or brown pigments (clusters E2, E7 and E17). According to the taxonomic keys in Bergey's Manual of Determinative Bacteriology (Weeks, 1974), these strains are included in the genus Flavobacterium, a heterogeneous genus defined primarily on pigment production and failure to show 'unusual' characteristics such as ring formation or plant pathogenicity which would place the organisms in genera such as Microcyclus and Xanthomonas.

4. Gram-negative, non-motile, non-fermentative, oxidase-position, catalase-negative rods which are not actively proteolytic in gelatin media (E6). These strains resembled non-motile strains of Alcaligenes except for the catalase test results (Holding \& Shewan, 1974).

5. Gram-positive, motile rods producing pink colonies and spherical bodies in older cultures (E3). The morphogenesis of these strains is representative of coryneform bacteria (e.g. Arthrobacter) which also exhibit a morphological cycle (Bousfield, 1978; Mulder, 1964; Keddie, 1974, 1978).

6. Gram-positive-Gram-variable, motile, large rods forming endospores and growing aerobically (E9). The strains in this cluster clearly belong in the genus Bacillus (Gordon et al., 1973). The endospores were oval, terminal or subterminal and did not distend the sporangium. According to the keys of Gordon et al. (1973), strains occurring in this cluster are 
Table 5. Sources of isolates in each cluster shown as the percentage isolated from each of the 12 sampling stations in the Northwest Gulf of Alaska

\begin{tabular}{|c|c|c|c|c|c|c|c|c|c|c|c|c|}
\hline $\begin{array}{l}\text { Cluster } \\
\text { No. of strains }\end{array}$ & $\begin{array}{r}W 1 \\
4\end{array}$ & $\begin{array}{r}\text { W2 } \\
3\end{array}$ & $\begin{array}{r}\text { W3 } \\
20\end{array}$ & $\begin{array}{r}\text { w4 } \\
3\end{array}$ & $\begin{array}{r}\text { W5 } \\
3\end{array}$ & $\begin{array}{r}\text { W6 } \\
3\end{array}$ & $\begin{array}{r}w 7 \\
5\end{array}$ & $\begin{array}{r}\text { w8 } \\
7\end{array}$ & $\begin{array}{r}\text { W9 } \\
9\end{array}$ & $\begin{array}{r}\text { W10 } \\
4\end{array}$ & $\begin{array}{r}\text { W11 } \\
3\end{array}$ & $\begin{array}{r}W 12 \\
3\end{array}$ \\
\hline Station & & & & & & & & & & & & \\
\hline 101 & - & 33 & 30 & - & - & - & - & 14 & - & 一 & - & - \\
\hline 106 & - & - & 5 & - & $\overline{22}$ & - & 一 & - & - & - & - & 一 \\
\hline 119 & - & - & 5 & - & 33 & - & - & - & 一 & 一 & - & - \\
\hline 121 & - & - & - & - & - & - & 20 & 14 & - & - & - & 一 \\
\hline 124 & 25 & 33 & - & 33 & - & - & - & 14 & - & - & - & - \\
\hline 133 & 25 & 33 & 25 & 33 & - & $\overline{0}$ & $\bar{\Omega}$ & $\overline{0}$ & 11 & - & - & - \\
\hline 134 & - & - & 一 & - & - & 33 & 80 & 29 & - & - & - & - \\
\hline 137 & 25 & - & 30 & 33 & 67 & - & - & - & 89 & 100 & - & 一 \\
\hline 145 & 25 & - & - & - & - & - & - & 14 & - & - & 33 & 一 \\
\hline 148 & - & - & 5 & - & - & 33 & 一 & - & 一 & 一 & - & - \\
\hline 156 & 一 & - & - & - & - & 33 & - & - & - & - & - & - \\
\hline 159 & - & - & - & - & - & - & - & 14 & - & - & 67 & 100 \\
\hline
\end{tabular}

assigned to the Group I Bacillus species. The strains exhibited denitrifying activities and showed eurytolerance to physiological growth conditions, growing over a wide range of $\mathrm{pH}$ values, salt concentrations and temperatures.

In addition to the organisms that formed defined major clusters, several minor clusters showed characteristics of the genera Flavobacterium (strains in E8 were Gram-negative, motile rods producing yellow pigments), Pseudomonas (strains in E5 were Gram-negative, motile rods that grew only oxidatively) and Vibrio (strains in E11, E12 and E16 were Gramnegative rods generally with a curved axis). Other clusters (E1 and E4) could not be associated with defined genera.

\section{Characteristics and identification of Northwest Gulf of Alaska isolates}

The bacterial populations in this region were quite diverse; only 12 clusters containing three or more members were found at the $70 \%$ similarity level and of these only four contained five or more strains. The larger clusters generally accommodated isolates from several locations (Table 5); the two largest clusters contained a mixture of isolates from water and sediment and from 5 and $20^{\circ} \mathrm{C}$ isolation temperatures. Only two of the clusters were restricted to isolates from a single location.

The feature frequencies of the 12 clusters are shown in Table 6. Most isolates were psychrotrophs capable of growth at 5 and $20^{\circ} \mathrm{C}$, the normal temperature range for the area sampled; all grew well at $3 \% \mathrm{NaCl}$ concentrations and several would not grow in the absence of added $\mathrm{NaCl}$. Four of the clusters (W6, W8, W9 and W10) contained strains which exhibited complex nutritional growth factor requirements; those of one cluster (W7) required vitamins; but those in the remainder showed no growth factor requirements (W1, W2, W3, W4, W5, W11 and W12).

None of the reference strains were recovered within the 12 clusters and none of the isolates were identified using the computer comparison with ATCC strains. Based on the features shown in Table 6, seven categories of clusters can be described, five of which show major characteristics of defined generic groups:

1. Gram-negative, oxidase-positive, non-pigmented, fermentative, motile rods (clusters W2 and W3). These organisms were similar to Beneckea species (Baumann et al., 1971).

2. Gram-negative, oxidase-positive, non-pigmented, variably fermentative, motile, curved or straight rods (clusters W5 and W7). The morphological and biochemical characteristics of these organisms closely resembled those of Vibrio species (Davis \& Park, 1962) or Beneckea species (Baumann et al., 1971). Members of these clusters were stenohaline growing only at $3 \% \mathrm{NaCl}$.

3. Gram-negative, facultatively anaerobic, straight rods producing non-diffusible yellow 
Table 6. Feature frequencies of selected characteristics of major clusters of bacterial populations isolated from the Northwest Gulf of Alaska*

Cluster

No. of strains

Cell morphology

Curved axis

Pleomorphic

Rounded ends

Square ends

Cell length

$0.5-1.0 \mu \mathrm{m}$

$1 \cdot 1-2 \cdot 0 \mu \mathrm{m}$

2.1-3.0 $\mu \mathrm{m}$

Cell width

$<0.5 \mu \mathrm{m}$

$0.5-1.0 \mu \mathrm{m}$

$1 \cdot 1-2 \cdot 0 \mu \mathrm{m}$

Miscellaneous cell features

PHB $†$ inclusions

Capsule

Sudan black

Gram-negative

Motile

Colony pigmentation

Diffusible yellow

Non-diffusible violet

Non-diffusible yellow

Grey (no pigment)

Colony size

$<1 \mathrm{~mm}$

1-2 mm

2-6 mm

Colony morphology

Translucent

Transparent

Opaque

Entire

Undulate

Raised

Umbonate

Convex

Mucoid

Acid from

D-Ribose

D-Fructose

Cellobiose

Lactose

Sucrose

D-Mannitol

D-Glucose (aerobic)

D-Glucose (fermentative)

Miscellaneous features

Obligate aerobe

Facultative anaerobe

Indole

Ammonia produced

Nitrate reduced

Nitrite reduced

Starch hydrolysis $\begin{array}{rrrrrrrrrrrr}\text { W1 } & \text { W2 } & \text { W3 } & \text { W4 } & \text { W5 } & \text { W6 } & \text { W7 } & \text { W8 } & \text { W9 } & \text { W10 } & \text { W11 } & \text { W12 } \\ 4 & 3 & 20 & 3 & 3 & 3 & 5 & 7 & 9 & 4 & 3 & 3\end{array}$

$\begin{array}{rrrrrrrrrrrr}50 & 0 & 0 & 0 & 33 & 0 & 60 & 57 & 0 & 0 & 66 & 100 \\ 0 & 0 & 0 & 0 & 0 & 0 & 20 & 14 & 0 & 0 & 0 & 0 \\ 100 & 100 & 94 & 100 & 100 & 100 & 100 & 71 & 100 & 100 & 100 & 100 \\ 0 & 0 & 5 & 0 & 0 & 0 & 0 & 28 & 0 & 0 & 0 & 0 \\ & & & & & & & & & & & \\ 0 & 0 & 10 & 0 & 0 & 0 & 20 & 0 & 100 & 0 & 0 & 100 \\ 75 & 33 & 78 & 100 & 66 & 0 & 60 & 85 & 0 & 100 & 66 & 0 \\ 25 & 66 & 10 & 0 & 33 & 100 & 20 & 14 & 0 & 0 & 33 & 0\end{array}$

$\begin{array}{rrrrrrrrrrrr}0 & 0 & 5 & 0 & 0 & 0 & 0 & 0 & 0 & 0 & 0 & 100 \\ 100 & 100 & 89 & 100 & 100 & 100 & 100 & 100 & 100 & 100 & 100 & 0 \\ 0 & 0 & 5 & 0 & 0 & 0 & 0 & 0 & 0 & 0 & 0 & 0\end{array}$

$\begin{array}{rrrrrrrrrrrr}0 & 0 & 5 & 0 & 0 & 66 & 0 & 42 & 0 & 0 & 0 & 0 \\ 25 & 33 & 15 & 33 & 33 & 33 & 40 & 57 & 100 & 100 & 0 & 0 \\ 0 & 0 & 26 & 0 & 0 & 33 & 0 & 14 & 0 & 0 & 0 & 0 \\ 100 & 100 & 100 & 100 & 100 & 100 & 100 & 85 & 100 & 100 & 100 & 100 \\ 100 & 66 & 78 & 100 & 100 & 66 & 20 & 42 & 0 & 0 & 0 & 0\end{array}$

$\begin{array}{rrrrrrrrrrrr}0 & 0 & 0 & 0 & 0 & 0 & 0 & 14 & 0 & 0 & 0 & 0 \\ 0 & 0 & 0 & 66 & 0 & 0 & 0 & 0 & 0 & 0 & 0 & 0 \\ 0 & 0 & 10 & 0 & 0 & 0 & 0 & 0 & 100 & 100 & 100 & 100 \\ 100 & 100 & 89 & 33 & 100 & 100 & 100 & 100 & 0 & 0 & 0 & 0\end{array}$

$\begin{array}{rrrrrrrrrrrr}0 & 0 & 0 & 0 & 0 & 0 & 0 & 0 & 22 & 100 & 0 & 0 \\ 0 & 0 & 5 & 0 & 0 & 66 & 80 & 85 & 77 & 0 & 66 & 100 \\ 100 & 100 & 94 & 100 & 100 & 33 & 20 & 14 & 0 & 0 & 33 & 0\end{array}$

$\begin{array}{llllllllllll}50 & 100 & 89 & 0 & 66 & 100 & 100 & 71 & 0 & 0 & 100 & 100\end{array}$

$\begin{array}{rrrrrrrrrrrr}50 & 0 & 0 & 0 & 0 & 0 & 0 & 0 & 0 & 0 & 0 & 0 \\ 0 & 0 & 10 & 100 & 33 & 0 & 0 & 28 & 100 & 100 & 0 & 0\end{array}$

$\begin{array}{rrrrrrrrrrrr}75 & 0 & 89 & 100 & 100 & 100 & 100 & 100 & 100 & 100 & 100 & 100\end{array}$

$\begin{array}{llllllllllll}25 & 100 & 10 & 0 & 0 & 0 & 0 & 0 & 0 & 0 & 0 & 0\end{array}$

$\begin{array}{rrrrrrrrrrrr}100 & 100 & 63 & 33 & 33 & 66 & 100 & 100 & 0 & 0 & 100 & 100\end{array}$

$\begin{array}{llllllllllll}0 & 0 & 31 & 66 & 66 & 0 & 0 & 0 & 0 & 0 & 0 & 0\end{array}$

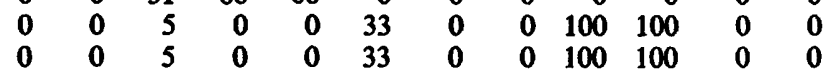

$\begin{array}{rrrrrlrlllll}0 & - & 92 & 50 & 50 & - & 50 & 0 & 0 & 0 & 0 & 0 \\ - & 100 & 94 & 50 & 50 & - & 0 & 0 & 0 & 0 & - & - \\ 0 & 0 & 22 & 0 & 0 & - & 60 & 0 & 0 & 0 & - & - \\ 0 & 0 & 10 & 0 & 0 & - & 66 & - & 0 & 0 & - & - \\ 0 & 0 & 0 & 50 & 33 & - & 0 & 0 & 0 & 0 & - & - \\ 0 & 100 & 94 & 100 & 66 & - & 0 & 0 & 0 & 0 & - & 100 \\ 0 & 100 & 94 & 50 & 33 & - & 100 & 0 & 0 & 0 & 0 & 0 \\ 50 & 100 & 88 & 100 & 66 & 0 & 100 & 0 & 0 & 25 & 66 & 0\end{array}$

$\begin{array}{llllllllllll}100 & 0 & 5 & 0 & 66 & 50 & 0 & 0 & 0 & 0 & 0 & 33\end{array}$

$\begin{array}{llllllllllll}0 & 100 & 95 & 100 & 33 & 50 & 100 & 100 & 100 & 100 & 100 & 66\end{array}$

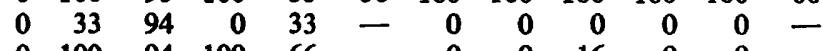

$\begin{array}{llllllllllll}0 & 100 & 94 & 100 & 66 & - & 0 & 0 & 16 & 0 & 0 & -\end{array}$

$\begin{array}{llllllllllll}0 & 0 & 95 & 100 & 66 & 0 & 100 & 66 & 75 & 50 & 0 & 0\end{array}$

$\begin{array}{rrrrrrrrrrrr}0 & 0 & 0 & 50 & 0 & 0 & 0 & 16 & 0 & 0 & 0 & 0 \\ 0 & 100 & 90 & 100 & 0 & 66 & 0 & 0 & 75 & 75 & & 100\end{array}$ 
Table 6. (cont.)

Cluster

No. of strains

Tween 20 hydrolysis

Tween 80 hydrolysis

L-Arginine decarboxylase

L-Lysine decarboxylase

L-Ornithine decarboxylase

Alkaline phosphatase

Catalase

Oxidase

$\mathrm{pH}$ : growth at

pH 6

pH 8

pH 9

pH 10

Temperature: growth at $5^{\circ} \mathrm{C}$

$20^{\circ} \mathrm{C}$

$25^{\circ} \mathrm{C}$

$37^{\circ} \mathrm{C}$

$\mathrm{NaCl}$ : growth at

$0.5 \%(\mathrm{w} / \mathrm{v})$

$5.0 \%(w / v)$

$7.5 \%(w / v)$

Carbohydrates utilized

D-Ribose

D-Fructose

D-Galactose

D-Glucose

D-Mannose

L-Sorbose

Salicin

Cellobiose

Lactose

Maltose

Sucrose

Trehalose

Alcohols utilized

1-Propanol

Glycerol

D-Mannitol

D-Sorbitol

meso-Inositol

2-Phenyl ethanol

Carboxylic acids utilized

Acetic acid

Caproic acid

Lauric acid

Propionic acid

Glutaric acid

Malonic acid

Succinic acid

Oleic acid

Itaconic acid

DL-Glyceric acid

$\beta$-Hydroxybutyric acid DL-Lactic acid

L(t)-Tartaric acid

Citric acid

$\begin{array}{rrrrrrrrrrrr}\text { W1 } & \text { W2 } & \text { W3 } & \text { W4 } & \text { W5 } & \text { W6 } & \text { W7 } & \text { W8 } & \text { W9 } & \text { W10 } & \text { W11 } & \text { W12 } \\ 4 & 3 & 20 & 3 & 3 & 3 & 5 & 7 & 9 & 4 & 3 & 3 \\ 75 & 66 & 95 & 100 & 0 & 100 & 0 & 50 & 0 & 0 & 0 & 0 \\ 100 & 66 & 95 & 100 & - & 100 & 0 & 0 & 0 & 0 & - & 0 \\ 0 & 33 & 65 & 0 & 0 & 0 & 100 & 83 & 25 & 50 & 50 & - \\ 0 & 0 & 0 & 0 & 0 & 0 & 66 & 33 & 0 & 0 & 0 & - \\ 0 & 0 & 0 & 0 & 0 & 0 & 66 & 25 & 0 & 25 & 0 & - \\ 100 & 100 & 90 & 50 & 100 & 100 & 100 & 100 & 71 & - & 100 & 100 \\ 0 & 100 & 100 & 50 & 100 & 0 & 80 & 85 & 33 & 75 & 100 & 100 \\ 50 & 100 & 100 & 100 & 100 & 100 & 100 & 100 & 11 & 0 & 0 & 33\end{array}$

$\begin{array}{rrrrrrrrrrrr}\mathbf{0} & \mathbf{0} & \mathbf{7 8} & \mathbf{0} & \mathbf{0} & \mathbf{0} & \mathbf{0} & \mathbf{0} & \mathbf{1 1} & \mathbf{0} & \mathbf{0} & \mathbf{0} \\ 100 & 100 & 85 & 100 & 100 & 100 & 100 & 100 & \mathbf{6 6} & 100 & \mathbf{6 6} & 100 \\ 100 & 100 & 90 & 100 & 100 & 100 & 100 & 71 & 11 & 25 & 0 & 0 \\ 100 & 100 & 95 & 100 & 0 & 0 & 0 & 28 & 0 & 0 & 0 & 0\end{array}$

$\begin{array}{llllllllllll}100 & 100 & 100 & 100 & - & 100 & 100 & 100 & 77 & 100 & 100 & 100\end{array}$

$\begin{array}{rrrrrrrrrrrr}100 & 100 & 100 & 50 & 100 & 100 & 80 & 42 & 100 & 75 & 100 & 100\end{array}$

$\begin{array}{rrrrrrrrrrrr}0 & 100 & 100 & 0 & 100 & 0 & 40 & 0 & 0 & 0 & 0 & 0 \\ 0 & 0 & 10 & 0 & 0 & 0 & 20 & 0 & 0 & - & - & -\end{array}$

$\begin{array}{rrrrrrrrrrrr}0 & 33 & 100 & 50 & 0 & 0 & 0 & 0 & 0 & 0 & 0 & 0 \\ 100 & 100 & 75 & 50 & 100 & 0 & 100 & 42 & 11 & 0 & 0 & 0 \\ 0 & 0 & 85 & 0 & 0 & 0 & 0 & 0 & 0 & 0 & 0 & 0\end{array}$

\begin{tabular}{|c|c|c|c|c|c|}
\hline 0 & 100 & 0 & 0 & & \\
\hline $\begin{array}{ll}0 & 100\end{array}$ & 100 & $\mathbf{0}$ & 0 & & \\
\hline $\begin{array}{ll}0 & 100\end{array}$ & 42 & o & o & & \\
\hline 100 & 100 & 0 & o & & \\
\hline 100 & 95 & 0 & 0 & & \\
\hline 66 & 0 & 0 & . & & \\
\hline 66 & 10 & 50 & 0 & & \\
\hline 33 & 20 & 0 & 33 & & \\
\hline 0 & 0 & 50 & 33 & & \\
\hline 0 & 5 & 0 & 0 & & \\
\hline 66 & 5 & 100 & 33 & & \\
\hline 100 & 100 & 0 & 0 & & \\
\hline
\end{tabular}

$\begin{array}{rrrrrrrrrrrr}\mathbf{0} & 100 & \mathbf{0} & \mathbf{5 0} & \mathbf{0} & \mathbf{0} & \mathbf{0} & \mathbf{0} & \mathbf{0} & \mathbf{0} & \mathbf{0} & \mathbf{0} \\ 25 & 100 & \mathbf{1 0 0} & \mathbf{5 0} & \mathbf{0} & \mathbf{0} & \mathbf{0} & \mathbf{0} & \mathbf{0} & \mathbf{0} & \mathbf{0} & \mathbf{0} \\ \mathbf{0} & \mathbf{1 0 0} & 100 & \mathbf{0} & \mathbf{6 6} & \mathbf{0} & \mathbf{0} & \mathbf{0} & \mathbf{0} & \mathbf{0} & \mathbf{0} & \mathbf{0} \\ \mathbf{0} & 100 & \mathbf{0} & \mathbf{0} & \mathbf{0} & \mathbf{0} & \mathbf{0} & \mathbf{0} & \mathbf{0} & \mathbf{0} & \mathbf{0} & \mathbf{0} \\ \mathbf{0} & 33 & \mathbf{5} & \mathbf{0} & \mathbf{0} & \mathbf{0} & \mathbf{0} & \mathbf{0} & \mathbf{0} & \mathbf{0} & \mathbf{0} & \mathbf{0} \\ \mathbf{0} & 66 & \mathbf{0} & \mathbf{5 0} & \mathbf{0} & \mathbf{0} & \mathbf{0} & \mathbf{0} & \mathbf{0} & \mathbf{0} & \mathbf{0} & \mathbf{0}\end{array}$

$\begin{array}{rrrrrrrrrrrr}\mathbf{0} & 100 & 95 & 50 & 100 & 0 & 0 & 0 & 0 & 0 & 0 & 100 \\ 0 & 0 & 15 & 0 & 0 & 0 & 0 & 0 & 0 & 0 & 0 & 0 \\ 100 & 100 & 10 & 100 & 0 & 0 & 0 & 0 & 22 & 0 & 0 & 0 \\ 0 & 0 & 20 & 0 & 0 & 0 & 0 & 0 & 0 & 0 & 0 & 0 \\ 0 & 100 & 40 & 50 & 0 & 0 & 0 & 0 & 0 & 0 & 0 & 0 \\ 25 & 100 & 95 & 0 & 0 & 0 & 0 & 0 & 0 & 0 & 0 & 0 \\ 25 & 100 & 0 & 50 & 0 & 66 & 0 & 0 & 0 & 0 & 0 & 0 \\ 0 & 0 & 0 & 50 & 0 & 0 & 0 & 0 & 0 & 0 & 0 & 0 \\ 0 & 0 & 5 & 0 & 0 & 0 & 20 & 14 & 0 & 0 & 0 & 0 \\ 25 & 0 & 65 & 0 & 0 & 0 & 0 & 0 & 0 & 0 & 0 & 0 \\ 0 & 0 & 0 & 50 & 66 & 0 & 0 & 0 & 0 & 0 & 0 & 0 \\ 0 & 100 & 35 & 0 & 100 & 0 & 0 & 0 & 0 & 0 & 0 & 100 \\ 0 & 0 & 0 & 0 & 0 & 0 & 0 & 0 & 0 & 0 & 100 & 33 \\ 0 & 100 & 30 & 50 & 66 & 0 & 0 & 0 & 0 & 0 & 0 & 0\end{array}$


Table 6. (cont.)

Cluster

No. of strains

Pyruvic acid

2-Ketoglutaric acid

Ascorbic acid

Galacturonic acid

D-Gluconic acid

Stearic acid

Amino acids utilized

L-Alanine

L-Arginine

L-Asparagine

L-Aspartic acid

L-Cysteine

L-Cystine

L-Glutamic acid

Glycine

L-Leucine

L-Isoleucine

L-Ornithine

L-Phenylalanine

L-Proline

L-Serine

L-Threonine

L-Tryptophan

L-Tyrosine

L-Valine

Amines utilized

$\mathrm{N}$-Acetylglucosamine

Guanine

Thymine

Hydrocarbons utilized n-Pentadecane

1-Methylnaphthalene w-Phenyldecane

$\begin{array}{rrrrrrrrrrrr}\text { W1 } & \text { W2 } & \text { W3 } & \text { W4 } & \text { W5 } & \text { W6 } & \text { W7 } & \text { W8 } & \text { W9 } & \text { W10 } & \text { w11 W12 } \\ 4 & 3 & 20 & 3 & 3 & 3 & 5 & 7 & 9 & 4 & 3 & 3 \\ 75 & 100 & 95 & 50 & 100 & 0 & 0 & 0 & 0 & 0 & 100 & 33 \\ 0 & 100 & 90 & 50 & 100 & 0 & 40 & 0 & 0 & 0 & 66 & 100 \\ 0 & 0 & 65 & 0 & 0 & 0 & 0 & 0 & 0 & 0 & 0 & 0 \\ 0 & 0 & 0 & 50 & 0 & 0 & 0 & 0 & 0 & 0 & 0 & 0 \\ 0 & 100 & 15 & 0 & 0 & 0 & 0 & 0 & 0 & 0 & 0 & 0 \\ 0 & 0 & 0 & 0 & 0 & 0 & 0 & 0 & 25 & 0 & - & 0\end{array}$

$\begin{array}{rrrrrrrrrrrr}75 & 66 & 100 & 50 & 100 & 0 & 0 & 0 & 0 & 0 & 0 & 0 \\ 100 & 66 & 40 & 50 & 100 & 0 & 100 & 33 & 0 & 0 & - & 0 \\ 25 & 100 & 100 & 50 & 100 & 0 & 80 & 0 & 0 & 0 & 0 & 0 \\ 0 & 100 & 100 & 0 & 0 & 0 & 100 & 14 & 0 & 0 & 100 & 100 \\ 0 & 100 & 40 & 50 & 0 & 0 & 20 & 0 & 0 & 0 & 0 & 0 \\ 0 & 100 & 15 & 100 & 0 & 0 & 0 & 0 & 0 & 0 & 0 & 0 \\ 100 & 100 & 100 & 100 & 100 & 0 & 80 & 14 & 0 & 0 & 33 & 66 \\ 75 & 100 & 100 & 0 & 0 & 0 & 0 & 0 & 0 & 0 & 0 & 0 \\ 100 & 0 & 68 & 50 & 0 & 0 & 0 & 0 & 0 & 0 & - & 0 \\ 0 & 0 & 15 & 50 & 66 & 0 & 0 & 0 & 0 & 0 & - & 0 \\ 0 & 66 & 15 & 50 & 100 & 0 & 0 & 0 & 0 & 0 & 0 & 0 \\ 0 & 0 & 10 & 50 & 100 & 0 & 0 & 0 & 0 & 0 & - & 0 \\ 100 & 100 & 100 & 100 & 100 & 0 & 100 & 14 & 0 & 0 & 0 & 0 \\ 100 & 100 & 100 & 0 & 100 & 0 & 0 & 0 & 0 & 0 & 0 & 0 \\ 0 & 100 & 95 & 0 & 0 & 0 & 20 & 0 & 0 & 0 & 0 & 0 \\ 0 & 0 & 5 & 0 & 0 & 0 & 0 & 0 & 0 & 0 & 0 & 0 \\ 100 & 33 & 90 & 100 & 100 & 0 & 0 & 0 & 0 & 0 & 0 & 0 \\ 0 & - & 25 & 100 & 0 & - & 0 & 0 & 0 & 0 & 0 & 0\end{array}$

$\begin{array}{rrrrrrrrrrrr}100 & 100 & 100 & 0 & 100 & 0 & 40 & 0 & 0 & 0 & 0 & 0 \\ 0 & 33 & 0 & 50 & 0 & 0 & 0 & 0 & 0 & 0 & 0 & 33 \\ 0 & 100 & 0 & 50 & 0 & 0 & 0 & 0 & 0 & 0 & 0 & 0\end{array}$

$\begin{array}{llllllllllll}\mathbf{0} & \mathbf{0} & 25 & \mathbf{0} & \mathbf{0} & \mathbf{0} & \mathbf{0} & \mathbf{0} & \mathbf{0} & \mathbf{0} & \mathbf{0} & \mathbf{0} \\ \mathbf{0} & \mathbf{0} & \mathbf{2 5} & \mathbf{0} & \mathbf{0} & \mathbf{0} & \mathbf{0} & \mathbf{0} & \mathbf{0} & \mathbf{0} & \mathbf{0} & \mathbf{0} \\ \mathbf{0} & \mathbf{0} & \mathbf{1 0} & \mathbf{0} & \mathbf{0} & \mathbf{0} & \mathbf{0} & \mathbf{0} & \mathbf{0} & \mathbf{0} & \mathbf{0} & \mathbf{0}\end{array}$

* The following substrates were not used by strains in any of the clusters: L-arabinose, D-xylose, raffinose, L-rhamnose, 1-butanol, ethanol, 2-propanol, D-arabitol, dulcitol, phenol, caprylic acid, fumaric acid, benzoic acid, $\alpha$-aminobutyric acid, L-lysine, L-methionine, ethanolamine, histamine, putrescine, 2-methylnaphthalene. The following features were $100 \%$ positive for all strains: rod-shaped, predominance of single cells, glistening colonies, smooth colonies, growth at $\mathrm{pH} 7$, growth at $10^{\circ} \mathrm{C}$, growth at $15^{\circ} \mathrm{C}$ and growth at $3.0 \div \mathrm{NaCl}$.

+ PHB, Poly- $\beta$-hydroxybutyric acid.

pigments (clusters W9 and W10). According to taxonomic keys in Bergey's Manual of Determinative Bacteriology (Weeks, 1974), strains in these clusters are classified in the genus Flavobacterium. Members of these clusters were fastidious; they were restricted to growth at a salt concentration of $3 \% \mathrm{NaCl}$ and required complex media for growth.

4. Gram-negative, yellow-pigmented, non-motile rods forming partial rings (clusters W11 and W12). These organisms were morphologically similar to members of the genus Microcyclus (Claus et al., 1968; Raj, 1977; Staley, 1974). The strains were oxidase-negative and grew on a very limited number of substrates.

5. Gram-negative, oxidase-positive rods producing violet pigments (cluster W4). Isolates within this cluster produced violet pigments which were soluble in ethanol, but not water, and gave an absorption maximum at $579 \mathrm{~nm}$ characteristic of the pigment violacein produced by strains of the genus Chromobacterium (Sneath, 1956, 1974). The strains were motile and lost their pigment-producing ability on repeated subculturing.

6. Gram-negative, straight or curved rods that are catalase-negative (clusters W1 and 
W6). These strains have not been identified. Members of cluster W1 were non-fermentative; those of cluster W6 failed to grow in fermentation tests and thus could not be scored for these features.

7. Gram-negative, non-pigmented, pleomorphic rods exhibiting bipolar inclusions (cluster W8). These bacteria have not been identified. They showed a high degree of morphological variability and were nutritionally fastidious.

In addition to the organisms that were recovered in defined clusters, several individual organisms showed characteristics of coryneform bacteria (large rods forming spherical bodies in older culture) and several others were Gram-positive cocci which exhibited characteristics of the genus Micrococcus (Gram-positive cocci producing pigments and occurring singly or in pairs). Numerous other organisms were observed which were not readily associated with previously described taxa.

\section{DISCUSSION}

The dominant Moraxella-Acinetobacter populations found in the Northeast Gulf of Alaska were absent from the Northwest Gulf of Alaska. Indeed, while Acinetobacter and Moraxella strains are readily isolated from marine habitats, they have not previously been found as dominant marine bacterial populations. Some caution must be used in comparing and drawing firm conclusions about regional differences in species compositions in Alaskan Outer Continental Shelf areas since samples were collected at different times in each area; caution should also be used when comparing the sizes of populations in these different studies since different numbers of isolates were used in each study.

As in the Beaufort Sea (Kaneko et al., 1979), there were several clusters that were equated with Vibrio-Beneckea species and there was a notable lack of Pseudomonas strains in both the Northeast and Northwest Gulf regions. Vibrio-Beneckea species, which are commonly found in marine ecosystems (Kaneko \& Colwell, 1973, 1974), were frequently identified in this study. The repeated failure to find dominant populations of Pseudomonas is not likely to be an artefact of isolation procedures since, using the same basic medium marine agar 2216, ZoBell and colleagues (ZoBell \& Upham, 1944; ZoBell, 1946) isolated numerous Pseudomonas species in the Pacific between Hawaii and California. Simidu et al. (1977) also isolated Pseudomonas species from the Pacific Ocean.

A general feature of the isolates from the Northeast Gulf of Alaska is their eurytolerance to temperature, salt and $\mathrm{pH}$; most isolates grew at 5 to $37{ }^{\circ} \mathrm{C}, 0.5$ to $10 \% \mathrm{NaCl}$ and $\mathrm{pH}$ values of 6 to 10. There was a high incidence of diverse pigmented bacteria (44\% of total isolates) in this region, many of which formed single-membered clusters; this finding is similar to our observations in other Alaskan Continental Shelf regions. Some are undoubtedly flavobacteria, but some of these pigmented bacteria may be coryneforms (Keddie, 1978); difficulties in identifying marine members of the coryneform group have been discussed by Bousfield (1978). There appears to be a spatial discontinuity in the distribution of Microcyclus species; this genus is common to the contiguous region of the Northwest Gulf of Alaska, Bering Sea and Arctic Ocean, but not the Northeast Gulf of Alaska.

A surprisingly high proportion of the isolates from the Northwest Gulf of Alaska appear to be 'true marine bacteria' as shown by their salt requirements. Most of the organisms isolated from this region possess the features of autochthonous marine micro-organisms. Most isolates are psychrotrophs capable of growth over the full range of temperatures that occur in surface and bottom water layers of these habitats. No obligate psychrophiles were found. There is no universal definition for a true marine bacterium. ZoBell $(1961,1963)$ considers a marine bacterium to be one which, on initial isolation, grows in full-strength seawater $(3 \because \mathrm{NaCl})$, but not at one-quarter to one-third the osmotic pressure of seawater. Bacteria which, on initial isolation, are restricted to growth at or near $3 \% \mathrm{NaCl}$ and which grow in the range of 0 to $20^{\circ} \mathrm{C}$ were considered to meet the requirements of true marine 
bacteria. Other eurytolerant bacteria exist quite well in marine ecosystems as evidenced by the large number of such organisms isolated, but it is virtually impossible to classify such bacteria as truly marine since they could also exist in terrestrial and freshwater ecosystems. It is best to consider such organisms, which are able to grow in seawater under ambient conditions and which are isolated from marine ecosystems, as indigenous marine bacteria; they are functionally marine bacteria if not indeed true marine bacteria.

There was a lack of a dominant population in the Northwest Gulf of Alaska. Rather, the populations in this region were very diverse and most organisms formed single-membered clusters. There was a relatively high mortality rate among these original isolates as evidenced by the reduction from the original 25 isolates from each station to those used in the analyses which are shown in Table 1. We attribute this loss of viability in part to the complex nutritional requirements shown by many of the isolates which may not have been met during subculturing. The more stringent nutritional requirements of the Northwest Gulf isolates, compared with the Northeast Gulf isolates, are interesting to note; they probably reflect regional differences in nutrient availability.

Many of the pigmented isolates, which comprised $37 \%$ of the strains included in the cluster analysis, formed single-membered clusters. The incidence of pigmented bacteria in the Northwest Gulf of Alaska was somewhat lower than in the Northeast Gulf and higher than has been reported for some temperate marine waters (Lovelace et al., 1967). Microcyclus species were tentatively identified, based on morphology, near the Aleutian Islands; they have also been found in the Beaufort Sea (Kaneko et al., 1979) and Bering Sea (unpublished data) but not in the Northeast Gulf of Alaska.

The strains examined in this study show a heterogeneity of features that does not permit a clear separation of genera based on the use of individual 'key' phenotypic features; this is evidenced by the spectrum of features found for isolates that appear to be MoraxellaAcinetobacter species. Problems with relying on a single feature for determining taxonomic status are illustrated in this study, e.g. the reliance on yellow-orange pigmentation for classification of Flavobacterium species and on a negative oxidase test for classification of Acinetobacter.

The results of the catalase tests present a particular problem in classifying several phenetic groups in this study. The only Gram-negative, catalase-negative strains described in Bergey's Manual of Determinative Bacteriology are included in the genus Derxia (Becking, 1974). While the relationship of strains isolated from the Gulf of Alaska to the genus Derxia cannot be positively excluded, the features of the catalase-negative strains from the Gulf of Alaska do not resemble those in the description of Derxia gummosa. The catalase-negative isolates could represent a new group of generic rank, but it is not possible to make this conclusion based largely on this phenotypic feature which may be of dubious taxonomic value.

This paper highlights the difficulties in classifying bacteria from diverse habitats, which have been poorly studied, even when a large number of phenotypic features are considered. Many of the isolates examined in this study could not be identified.

The failure to recover reference strains within phenetic clusters makes identification of the Gulf of Alaska isolates difficult. The reference strains were selected because they represent genera normally reported for marine ecosystems. Most of the ATCC strains selected as reference strains were originally isolated from marine ecosystems, many from the Pacific Ocean near the Gulf of Alaska regions sampled in this study. In retrospect, it is not surprising that ATCC reference strains from temperate regions of the Pacific Ocean would not be included in phenetic clusters containing strains isolated from sub-Arctic Gulf of Alaska ecosystems. Most of the ATCC strains included in constructing the probabilistic identification matrices are not marine isolates and would not be expected to show a high similarity with marine isolates from the Gulf of Alaska. Species distribution appears to be quite different between the Northeast and Northwest Gulf of Alaska regions as well as between 
these regions and elsewhere in the Pacific Ocean and in other Alaskan Continental Shelf regions. Many new 'species' or genera probably exist within the Gulf of Alaska and other northern marine ecosystems.

This study was supported by the Bureau of Land Management through interagency agreement with the National Oceanic and Atmospheric Administration under which a multi-year programme, responding to needs of petroleum development of the Alaskan continental shelf, is managed by the Outer Continental Shelf Environmental Assessment Program (OCSEAP) Office. We wish to thank C. Pennington, K. Jabarizadeh and C. Short for laboratory assistance, E. J. Krichevsky for performing the computer manipulations and C. A. Walczak for assistance with the computer programs.

\section{REFERENCES}

Baumann, P., Doudoroff, M. \& Stanier, R. Y. (1968). A study of the Moraxella group. II. Oxidase-negative species (genus Acinetobacter). Journal of Bacteriology 95, 1520-1541.

Baumann, P., Baumann, L. \& Mandel, M. (1971). Taxonomy of marine bacteria: the genus Beneckea. Journal of Bacteriology 107, 268-294.

Becking, J. H. (1974). Genus Derxia. In Bergey's Manual of Determinative Bacteriology, 8th edn, pp. 260-261. Edited by R. E. Buchanan \& N. E. Gibbons. Baltimore: Williams \& Wilkins.

BousfiELd, I. J. (1978). The taxonomy of coryneform bacteria from the marine environment. In Coryneform Bacteria, pp. 217-234. Edited by I. J. Bousfield \& A. G. Callely. London: Academic Press.

Buchanan, R. E. \& Gibbons, N. E. (editors) (1974). Bergey's Manual of Determinative Bacteriology, 8th edn. Baltimore: Williams \& Wilkins.

Claus, D., Bergendahl, J. E. \& Mandel, M. (1968). DNA base composition of Microcyclus species and organisms of similar morphology. Archiv für Mikrobiologie 63, 26-68.

Davis, H. G. \& Park, R. W. A. (1962). A taxonomic study of certain bacteria currently classified as Vibrio species. Journal of General Microbiology 27, 101-119.

Gordon, R. E., Haynes, W. C. \& Pang, C. H.-N. (1973). The genus Bacillus. U.S. Department of Agriculture Handbook no. 427.

Holding, A. J. \& ShEWAN, J. M. (1974). Genera of uncertain affiliation; genus Alcaligenes. In Bergey's Manual of Determinative Bacteriology, 8th edn, pp. 273-277. Edited by R. E. Buchanan \& N. E. Gibbons. Baltimore: Williams \& Wilkins.

JoHNSON, R. (1979). Computer-aided identification. FDA By-Lines 9, 235-250.

Kaneko, T. \& Colwell, R. R. (1973). Ecology of Vibrio parahaemolyticus in Chesapeake Bay. Journal of Bacteriology 113, 24-32.

Kaneko, T. \& Colwell, R. R. (1974). Distribution of Vibrio parahaemolyticus and related organisms in the Atlantic Ocean off South Carolina and Georgia. Applied Microbiology 28, 1009-1017.

Kanfko, T., KRicheVsky, M. I. \& Atlas, R. M. (1979). Numerical taxonomy of bacteria from the Beaufort Sea. Journal of General Microbiology 110, 111-125.
KEDDIE, R. M. (1974). Genus Arthrobacter. In Bergey's Manual of Determinative Bacteriology, 8th edn, pp. 618-625. Edited by R. E. Buchanan \& N. E. Gibbons. Baltimore: Williams \& Wilkins.

KEDDIE, R. M. (1978). What do we mean by coryneform bacteria? In Coryneform Bacteria, pp. 1-12. Edited by I. J. Bousfield \& A. G. Callely. London: Academic Press.

LAUTROP, H. (1974). Genus Moraxella. Genus Acinetobacter. In Bergey's Manual of Determinative Bacteriology, 8th edn, pp. 433-438. Edited by R. E. Buchanan \& N. E. Gibbons. Baltimore: Williams \& Wilkins.

Lovelace, T. E., Tubiash, H. \& Colwell, R. R. (1967). Quantitative and qualitative commercial bacterial flora of Crassostrea virginica in Chesapeake Bay. Proceedings of the National Shellfisheries Association 58, 82-87.

Mulder, E. G. (1964). Arthrobacter. In Principle and Application in Aquatic Microbiology, Proceedings of Rudolfs Research Conference, pp. 254 279. Edited by H. Heukelekian \& N. C. Dondero. New York: John Wiley.

RAJ, H. D. (1977). Microcyclus and related ringforming bacteria. CRC Critical Reviews in Microbiology 5, 243-269.

ShewAN, J. M. (1971). The microbiology of fish and fishery products. A progress report. Journal of Applied Bacteriology 34, 299-315.

Simidu, U., Kaneko, E. \& TAga, N. (1977). Microbiological studies of Tokyo Bay. Microbial Ecology 3, 173-191.

SKERMAN, V. B. D. (1967). A Guide to the Identification of the Genera of Bacteria. Baltimore: Williams \& Wilkins.

SNeath, P. H. A. (1956). Cultural and biochemical characteristics of the genus Chromobacterium. Journal of General Microbiology 15, 70-98.

SNEATH, P. H. A. (1957). The application of computers to taxonomy. Journal of General Microbiology 17, 201-206.

SNeATH, P. H. A. (1974). Genus Chromobacterium. In Bergey's Manual of Determinative Bacteriology, 8th edn, pp. 354-357. Edited by R. E. Buchanan \& N. E. Gibbons, Baltimore: Williams \& Wilkins.

Staley, J. T. (1974). Genus Microcyclus. In Bergey's Manual of Determinative Bacteriology, 8th edn, 
pp. 214-215. Edited by R. E. Buchanan \& N. E. ZoBeLL, C. E. (1961). Importance of microorganGibbons. Baltimore: Williams \& Wilkins.

THORNLEY, M. J. (1967). A taxonomic study of Acinetobacter and related genera. Journal of General Microbiology 49, 211-257.

WalczaK, C. A. (1979). Complex data analysis. FDA Bj-Lines 9, 251-253.

WEEKS, O. B. (1974). Genus Flavobacterium. In Bergey's Manual of Determinative Bacteriology, 8th edn, pp. 357-364. Edited by R. E. Buchanan \& N. E. Gibbons. Baltimore: Williams \& Wilkins.

ZoBell, C. E. (1946). Marine Microbiology. Waltham, Mass.: Chronica Botanica. isms in the sea. Proceedings of Low Temperature Microbiology Symposium, Cambden, N.J., pp. 107-132.

ZoBell, C. E. (1963). Domain of the marine microbiologist. In Symposium on Marine Microbiology, pp. 3-24. Edited by C. H. Oppenheimer. Springfield, Ill.: Charles Thomas.

ZoBell, C. E. \& UpHaM, H. C. (1944). A list of marine bacteria including description of sixty new species. Bulletin of the Scripps Institution of Oceanography (Technical Series) 5, 239-292. 